\title{
Zirconium Complexes of a Cross-Bridged Cyclam
}

\author{
Paul O'Connor*, David J. Berg*, and Brendan Twamley \\ Department of Chemistry, University of Victoria, P.O. Box 3065 \\ Victoria, British Columbia, Canada V8W 3V6
}

Supporting Information 
Figure S1. ORTEP Drawing of (CBC)Zr(O-2,6-C $\left.{ }_{6} \mathrm{H}_{3} \mathrm{Me}_{2}\right)_{2}, 5$.

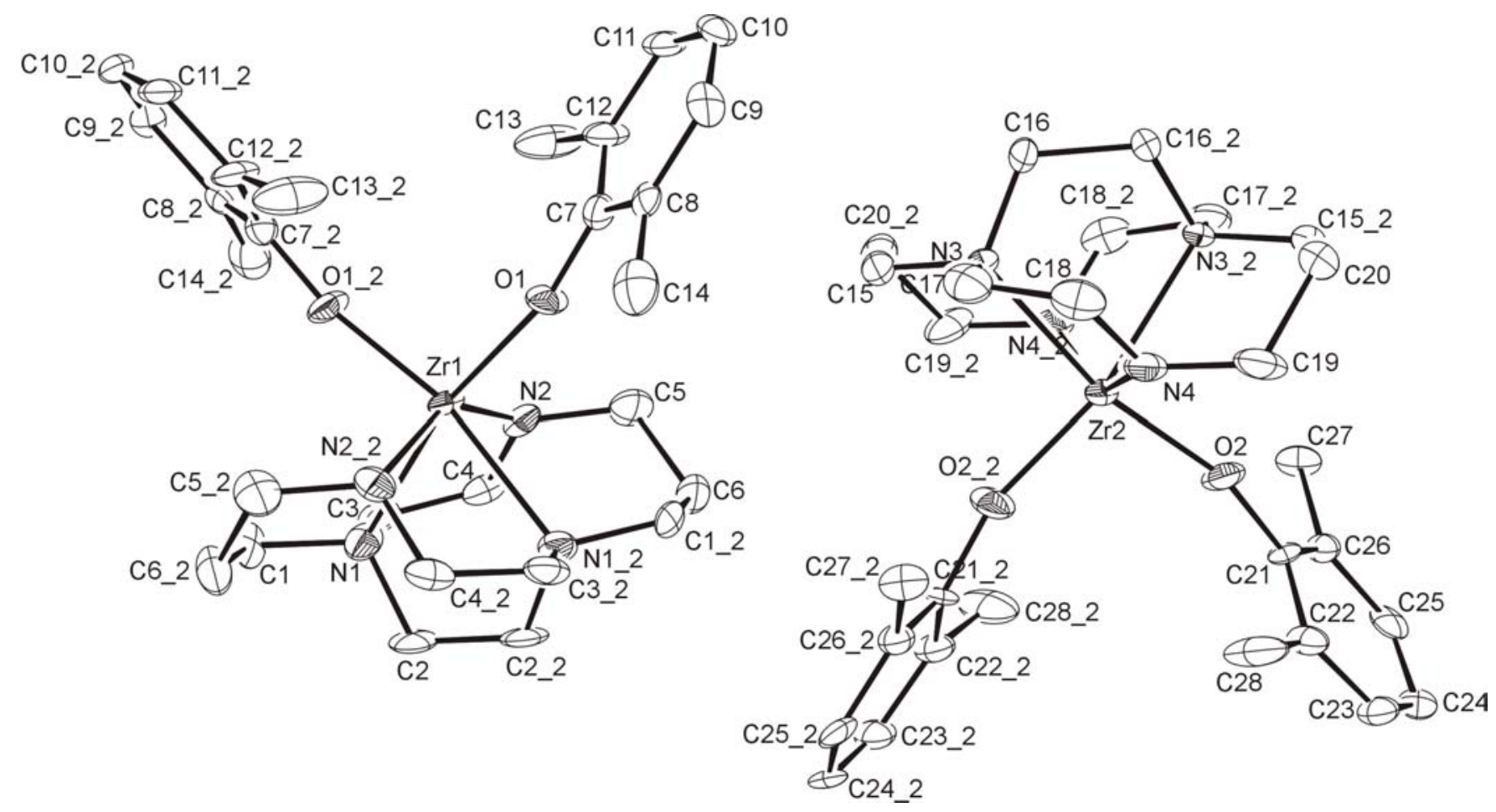


Table S1. Atomic coordinates $\left(\times 10^{4}\right)$ and equivalent isotropic displacement parameters $\left(\AA^{2} \times 10^{3}\right)$ for $(\mathrm{CBC}) \mathrm{Zr}\left(\mathrm{O}-2,6-\mathrm{C}_{6} \mathrm{H}_{3} \mathrm{Me}_{2}\right)_{2}, \mathbf{5}$.

\begin{tabular}{|c|c|c|c|c|}
\hline & $\mathrm{x}$ & $\mathrm{y}$ & $\mathrm{z}$ & $\mathrm{U}(\mathrm{eq})$ \\
\hline$C(1)$ & $11748(4)$ & $-1522(4)$ & $5262(3)$ & $30(1)$ \\
\hline$C(2)$ & $10502(5)$ & $-369(6)$ & $5998(3)$ & $31(1)$ \\
\hline$C(3)$ & $12037(3)$ & $512(4)$ & $5333(3)$ & $27(1)$ \\
\hline$C(4)$ & 11511(4) & $1651(5)$ & $5244(3)$ & 29(1) \\
\hline$C(5)$ & $9884(4)$ & $2543(5)$ & $4693(4)$ & $35(1)$ \\
\hline$C(6)$ & $9003(4)$ & 2567(4) & $5255(3)$ & $36(1)$ \\
\hline$C(7)$ & $8395(4)$ & $1029(4)$ & $3150(3)$ & $22(1)$ \\
\hline$C(8)$ & $7285(4)$ & $732(4)$ & $3017(3)$ & $20(1)$ \\
\hline $\mathrm{C}(9)$ & $6765(4)$ & $1205(4)$ & $2434(3)$ & $30(1)$ \\
\hline$C(10)$ & $7296(4)$ & 1935(4) & 2019(3) & $29(1)$ \\
\hline $\mathrm{C}(11)$ & $8423(4)$ & 2197(4) & $2136(3)$ & $27(1)$ \\
\hline$C(12)$ & $8980(4)$ & $1737(5)$ & $2708(3)$ & 27(1) \\
\hline$C(13)$ & $10200(5)$ & 1964(7) & 2803(4) & $49(2)$ \\
\hline$C(14)$ & $6707(6)$ & $-29(4)$ & $3491(5)$ & $39(2)$ \\
\hline$C(15)$ & $6766(4)$ & $3515(4)$ & $3721(3)$ & $34(1)$ \\
\hline$C(16)$ & $5448(4)$ & $4520(4)$ & $3026(3)$ & $25(1)$ \\
\hline$C(17)$ & $4826(4)$ & 2896(4) & $3700(3)$ & $33(1)$ \\
\hline$C(18)$ & $3623(4)$ & $3258(5)$ & $3801(3)$ & $36(1)$ \\
\hline$C(19)$ & $2474(4)$ & 4701(5) & $4324(3)$ & $36(1)$ \\
\hline$C(20)$ & $2361(4)$ & $5598(4)$ & $3742(3)$ & $39(1)$ \\
\hline
\end{tabular}




\begin{tabular}{|c|c|c|c|c|}
\hline $\mathrm{C}(21)$ & $3968(4)$ & $6638(4)$ & $5886(2)$ & $21(1)$ \\
\hline $\mathrm{C}(22)$ & $3246(4)$ & $6084(4)$ & $6364(3)$ & $24(1)$ \\
\hline $\mathrm{C}(23)$ & $2854(4)$ & $6722(4)$ & $6907(3)$ & $27(1)$ \\
\hline$C(24)$ & $3175(4)$ & $7790(4)$ & $7034(3)$ & $28(1)$ \\
\hline$C(25)$ & $3932(4)$ & $8307(4)$ & $6582(3)$ & $26(1)$ \\
\hline$C(26)$ & $4337(3)$ & $7729(4)$ & $6008(3)$ & $21(1)$ \\
\hline$C(27)$ & $5110(4)$ & $8315(5)$ & $5505(4)$ & $23(1)$ \\
\hline $\mathrm{C}(28)$ & $2938(7)$ & $4879(5)$ & $6255(4)$ & $45(2)$ \\
\hline $\mathrm{N}(1)$ & $11168(3)$ & $-401(4)$ & $5342(3)$ & $28(1)$ \\
\hline $\mathrm{N}(2)$ & $10642(3)$ & $1576(3)$ & $4710(3)$ & $28(1)$ \\
\hline $\mathrm{N}(3)$ & $5556(3)$ & $3888(3)$ & $3680(2)$ & $23(1)$ \\
\hline $\mathrm{N}(4)$ & $3559(3)$ & $4143(3)$ & $4327(2)$ & $26(1)$ \\
\hline $\mathrm{O}(1)$ & $8889(2)$ & $613(3)$ & $3710(2)$ & $27(1)$ \\
\hline $\mathrm{O}(2)$ & $4283(3)$ & $6069(3)$ & $5304(2)$ & $29(1)$ \\
\hline $\operatorname{Zr}(1)$ & 10000 & 0 & $4382(1)$ & $20(1)$ \\
\hline $\operatorname{Zr}(2)$ & 5000 & 5000 & $4649(1)$ & $21(1)$ \\
\hline
\end{tabular}

$\mathrm{U}(\mathrm{eq})$ is defined as one third of the trace of the orthogonalized $\mathrm{U}_{\mathrm{ij}}$ tensor. 
Table S2. Bond Lengths $(\AA)$ and Angles (deg) for $(\mathrm{CBC}) \mathrm{Zr}\left(\mathrm{O}-2,6-\mathrm{C}_{6} \mathrm{H}_{3} \mathrm{Me}_{2}\right)_{2}, \mathbf{5}$.

\begin{tabular}{|c|c|c|c|}
\hline \multicolumn{2}{|c|}{ Bond Lengths } & $\mathrm{C}(7)-\mathrm{O}(1)$ & $1.333(6)$ \\
\hline $\mathrm{C}(1)-\mathrm{N}(1)$ & $1.512(6)$ & $C(7)-C(12)$ & $1.392(7)$ \\
\hline$C(1)-C(6) \# 1$ & $1.533(7)$ & $\mathrm{C}(7)-\mathrm{C}(8)$ & $1.393(6)$ \\
\hline $\mathrm{C}(1)-\mathrm{H}(1 \mathrm{~A})$ & 0.9900 & $\mathrm{C}(8)-\mathrm{C}(9)$ & $1.409(6)$ \\
\hline $\mathrm{C}(1)-\mathrm{H}(1 \mathrm{~B})$ & 0.9900 & $C(8)-C(14)$ & $1.464(9)$ \\
\hline$C(2)-C(2) \# 1$ & $1.485(12)$ & $C(9)-C(10)$ & $1.344(8)$ \\
\hline $\mathrm{C}(2)-\mathrm{N}(1)$ & $1.502(7)$ & $\mathrm{C}(9)-\mathrm{H}(9)$ & 0.9500 \\
\hline $\mathrm{C}(2)-\mathrm{H}(2 \mathrm{~A})$ & 0.9900 & $C(10)-C(11)$ & $1.398(7)$ \\
\hline $\mathrm{C}(2)-\mathrm{H}(2 \mathrm{~B})$ & 0.9900 & $\mathrm{C}(10)-\mathrm{H}(10)$ & 0.9500 \\
\hline $\mathrm{C}(3)-\mathrm{N}(1)$ & $1.502(6)$ & $\mathrm{C}(11)-\mathrm{C}(12)$ & $1.405(7)$ \\
\hline$C(3)-C(4)$ & $1.504(7)$ & $\mathrm{C}(11)-\mathrm{H}(11)$ & 0.9500 \\
\hline $\mathrm{C}(3)-\mathrm{H}(3 \mathrm{~A})$ & 0.9900 & $\mathrm{C}(12)-\mathrm{C}(13)$ & $1.490(8)$ \\
\hline $\mathrm{C}(3)-\mathrm{H}(3 \mathrm{~B})$ & 0.9900 & $\mathrm{C}(13)-\mathrm{H}(13 \mathrm{~A})$ & 0.9800 \\
\hline$C(4)-N(2)$ & $1.469(6)$ & $\mathrm{C}(13)-\mathrm{H}(13 \mathrm{~B})$ & 0.9800 \\
\hline $\mathrm{C}(4)-\mathrm{H}(4 \mathrm{~A})$ & 0.9900 & $\mathrm{C}(13)-\mathrm{H}(13 \mathrm{C})$ & 0.9800 \\
\hline $\mathrm{C}(4)-\mathrm{H}(4 \mathrm{~B})$ & 0.9900 & $\mathrm{C}(14)-\mathrm{H}(14 \mathrm{~A})$ & 0.9800 \\
\hline $\mathrm{C}(5)-\mathrm{N}(2)$ & $1.464(7)$ & $\mathrm{C}(14)-\mathrm{H}(14 \mathrm{~B})$ & 0.9800 \\
\hline$C(5)-C(6)$ & $1.516(7)$ & $\mathrm{C}(14)-\mathrm{H}(14 \mathrm{C})$ & 0.9800 \\
\hline $\mathrm{C}(5)-\mathrm{H}(5 \mathrm{~A})$ & 0.9900 & $\mathrm{C}(15)-\mathrm{C}(20) \# 2$ & $1.484(7)$ \\
\hline $\mathrm{C}(5)-\mathrm{H}(5 \mathrm{~B})$ & 0.9900 & $\mathrm{C}(15)-\mathrm{N}(3)$ & $1.512(6)$ \\
\hline$C(6)-C(1) \# 1$ & $1.533(7)$ & $\mathrm{C}(15)-\mathrm{H}(15 \mathrm{~A})$ & 0.9900 \\
\hline $\mathrm{C}(6)-\mathrm{H}(6 \mathrm{~A})$ & 0.9900 & $\mathrm{C}(15)-\mathrm{H}(15 \mathrm{~B})$ & 0.9900 \\
\hline $\mathrm{C}(6)-\mathrm{H}(6 \mathrm{~B})$ & 0.9900 & $\mathrm{C}(16)-\mathrm{N}(3)$ & $1.482(7)$ \\
\hline
\end{tabular}




\begin{tabular}{|c|c|c|c|}
\hline $\mathrm{C}(16)-\mathrm{C}(16) \# 2$ & $1.565(9)$ & $C(24)-C(25)$ & $1.401(8)$ \\
\hline $\mathrm{C}(16)-\mathrm{H}(16 \mathrm{~A})$ & 0.9900 & $\mathrm{C}(24)-\mathrm{H}(24)$ & 0.9500 \\
\hline $\mathrm{C}(16)-\mathrm{H}(16 \mathrm{~B})$ & 0.9900 & $C(25)-C(26)$ & $1.397(7)$ \\
\hline $\mathrm{C}(17)-\mathrm{N}(3)$ & $1.469(7)$ & $\mathrm{C}(25)-\mathrm{H}(25)$ & 0.9500 \\
\hline $\mathrm{C}(17)-\mathrm{C}(18)$ & $1.510(7)$ & $C(26)-C(27)$ & $1.514(7)$ \\
\hline $\mathrm{C}(17)-\mathrm{H}(17 \mathrm{~A})$ & 0.9900 & $\mathrm{C}(27)-\mathrm{H}(27 \mathrm{~A})$ & 0.9800 \\
\hline $\mathrm{C}(17)-\mathrm{H}(17 \mathrm{~B})$ & 0.9900 & $\mathrm{C}(27)-\mathrm{H}(27 \mathrm{~B})$ & 0.9800 \\
\hline $\mathrm{C}(18)-\mathrm{N}(4)$ & $1.471(7)$ & $\mathrm{C}(27)-\mathrm{H}(27 \mathrm{C})$ & 0.9800 \\
\hline $\mathrm{C}(18)-\mathrm{H}(18 \mathrm{~A})$ & 0.9900 & $\mathrm{C}(28)-\mathrm{H}(28 \mathrm{~A})$ & 0.9800 \\
\hline $\mathrm{C}(18)-\mathrm{H}(18 \mathrm{~B})$ & 0.9900 & $\mathrm{C}(28)-\mathrm{H}(28 \mathrm{~B})$ & 0.9800 \\
\hline $\mathrm{C}(19)-\mathrm{N}(4)$ & $1.454(7)$ & $\mathrm{C}(28)-\mathrm{H}(28 \mathrm{C})$ & 0.9800 \\
\hline$C(19)-C(20)$ & $1.562(7)$ & $\mathrm{N}(1)-\operatorname{Zr}(1)$ & $2.377(4)$ \\
\hline $\mathrm{C}(19)-\mathrm{H}(19 \mathrm{~A})$ & 0.9900 & $\mathrm{~N}(2)-\operatorname{Zr}(1)$ & $2.125(4)$ \\
\hline C(19)-H(19B) & 0.9900 & $\mathrm{~N}(3)-\operatorname{Zr}(2)$ & $2.394(4)$ \\
\hline $\mathrm{C}(20)-\mathrm{C}(15) \# 2$ & $1.484(7)$ & $\mathrm{N}(4)-\operatorname{Zr}(2)$ & $2.094(4)$ \\
\hline $\mathrm{C}(20)-\mathrm{H}(20 \mathrm{~A})$ & 0.9900 & $\mathrm{O}(1)-\operatorname{Zr}(1)$ & $1.997(3)$ \\
\hline $\mathrm{C}(20)-\mathrm{H}(20 \mathrm{~B})$ & 0.9900 & $\mathrm{O}(2)-\mathrm{Zr}(2)$ & $1.994(4)$ \\
\hline $\mathrm{C}(21)-\mathrm{O}(2)$ & $1.370(5)$ & $\operatorname{Zr}(1)-\mathrm{O}(1) \# 1$ & $1.997(3)$ \\
\hline$C(21)-C(26)$ & $1.392(7)$ & $\mathrm{Zr}(1)-\mathrm{N}(2) \# 1$ & $2.125(4)$ \\
\hline$C(21)-C(22)$ & $1.428(7)$ & $\mathrm{Zr}(1)-\mathrm{N}(1) \# 1$ & $2.377(4)$ \\
\hline$C(22)-C(23)$ & $1.381(7)$ & $\mathrm{Zr}(2)-\mathrm{O}(2) \# 2$ & $1.994(4)$ \\
\hline$C(22)-C(28)$ & $1.498(8)$ & $\mathrm{Zr}(2)-\mathrm{N}(4) \# 2$ & $2.094(4)$ \\
\hline$C(23)-C(24)$ & $1.351(7)$ & $\mathrm{Zr}(2)-\mathrm{N}(3) \# 2$ & $2.394(4)$ \\
\hline C(23)-H(23) & 0.9500 & & \\
\hline
\end{tabular}




\section{Bond Angles}

$\mathrm{N}(1)-\mathrm{C}(1)-\mathrm{C}(6) \# 1 \quad 116.8(4)$

$\mathrm{N}(1)-\mathrm{C}(1)-\mathrm{H}(1 \mathrm{~A})$

$\mathrm{C}(6) \# 1-\mathrm{C}(1)-\mathrm{H}(1 \mathrm{~A})$

$\mathrm{N}(1)-\mathrm{C}(1)-\mathrm{H}(1 \mathrm{~B})$

$\mathrm{C}(6) \# 1-\mathrm{C}(1)-\mathrm{H}(1 \mathrm{~B})$

$\mathrm{H}(1 \mathrm{~A})-\mathrm{C}(1)-\mathrm{H}(1 \mathrm{~B})$

$\mathrm{C}(2) \# 1-\mathrm{C}(2)-\mathrm{N}(1)$

C(2)\#1-C(2)-H(2A)

$\mathrm{N}(1)-\mathrm{C}(2)-\mathrm{H}(2 \mathrm{~A})$

$\mathrm{C}(2) \# 1-\mathrm{C}(2)-\mathrm{H}(2 \mathrm{~B})$

$\mathrm{N}(1)-\mathrm{C}(2)-\mathrm{H}(2 \mathrm{~B})$

$\mathrm{H}(2 \mathrm{~A})-\mathrm{C}(2)-\mathrm{H}(2 \mathrm{~B})$

$\mathrm{N}(1)-\mathrm{C}(3)-\mathrm{C}(4)$

$\mathrm{N}(1)-\mathrm{C}(3)-\mathrm{H}(3 \mathrm{~A})$

$\mathrm{C}(4)-\mathrm{C}(3)-\mathrm{H}(3 \mathrm{~A})$

$\mathrm{N}(1)-\mathrm{C}(3)-\mathrm{H}(3 \mathrm{~B})$

$\mathrm{C}(4)-\mathrm{C}(3)-\mathrm{H}(3 \mathrm{~B})$

$\mathrm{H}(3 \mathrm{~A})-\mathrm{C}(3)-\mathrm{H}(3 \mathrm{~B})$

$\mathrm{N}(2)-\mathrm{C}(4)-\mathrm{C}(3)$

$\mathrm{N}(2)-\mathrm{C}(4)-\mathrm{H}(4 \mathrm{~A})$

$\mathrm{C}(3)-\mathrm{C}(4)-\mathrm{H}(4 \mathrm{~A})$

$\mathrm{N}(2)-\mathrm{C}(4)-\mathrm{H}(4 \mathrm{~B})$

$\mathrm{C}(3)-\mathrm{C}(4)-\mathrm{H}(4 \mathrm{~B})$
108.1

108.1

108.1

108.1

107.3

116.2(3)

108.2

108.2

108.2

108.2

107.4

$111.6(4)$

109.3

109.3

109.3

109.3

108.0

$108.6(4)$

110.0

110.0

110.0

110.0
H(4A)-C(4)-H(4B) 108.3

$\mathrm{N}(2)-\mathrm{C}(5)-\mathrm{C}(6) \quad 115.2(5)$

$\mathrm{N}(2)-\mathrm{C}(5)-\mathrm{H}(5 \mathrm{~A}) \quad 108.5$

$\mathrm{C}(6)-\mathrm{C}(5)-\mathrm{H}(5 \mathrm{~A}) \quad 108.5$

$\mathrm{N}(2)-\mathrm{C}(5)-\mathrm{H}(5 \mathrm{~B}) \quad 108.5$

$\mathrm{C}(6)-\mathrm{C}(5)-\mathrm{H}(5 \mathrm{~B}) \quad 108.5$

$\mathrm{H}(5 \mathrm{~A})-\mathrm{C}(5)-\mathrm{H}(5 \mathrm{~B}) \quad 107.5$

$\mathrm{C}(5)-\mathrm{C}(6)-\mathrm{C}(1) \# 1 \quad 113.3(5)$

$\mathrm{C}(5)-\mathrm{C}(6)-\mathrm{H}(6 \mathrm{~A}) \quad 108.9$

C(1)\#1-C(6)-H(6A) 108.9

$\mathrm{C}(5)-\mathrm{C}(6)-\mathrm{H}(6 \mathrm{~B}) \quad 108.9$

C(1)\#1-C(6)-H(6B) 108.9

H(6A)-C(6)-H(6B) 107.7

$\mathrm{O}(1)-\mathrm{C}(7)-\mathrm{C}(12) \quad 120.5(4)$

$\mathrm{O}(1)-\mathrm{C}(7)-\mathrm{C}(8) \quad 118.5(4)$

$\mathrm{C}(12)-\mathrm{C}(7)-\mathrm{C}(8) \quad 121.0(5)$

$\mathrm{C}(7)-\mathrm{C}(8)-\mathrm{C}(9) \quad 117.7(5)$

$\mathrm{C}(7)-\mathrm{C}(8)-\mathrm{C}(14) \quad 119.2(5)$

$\mathrm{C}(9)-\mathrm{C}(8)-\mathrm{C}(14)$

$\mathrm{C}(10)-\mathrm{C}(9)-\mathrm{C}(8) \quad 122.2(4)$

C(10)-C(9)-H(9) 118.9

$\mathrm{C}(8)-\mathrm{C}(9)-\mathrm{H}(9) \quad 118.9$

$\mathrm{C}(9)-\mathrm{C}(10)-\mathrm{C}(11) \quad 120.0(5)$

C(9)-C(10)-H(10) 120.0 


\begin{tabular}{|c|c|c|c|}
\hline $\mathrm{C}(11)-\mathrm{C}(10)-\mathrm{H}(10)$ & 120.0 & $\mathrm{H}(15 \mathrm{~A})-\mathrm{C}(15)-\mathrm{H}(15 \mathrm{~B})$ & 107.2 \\
\hline $\mathrm{C}(10)-\mathrm{C}(11)-\mathrm{C}(12)$ & $119.7(5)$ & $\mathrm{N}(3)-\mathrm{C}(16)-\mathrm{C}(16) \# 2$ & $115.5(3)$ \\
\hline $\mathrm{C}(10)-\mathrm{C}(11)-\mathrm{H}(11)$ & 120.1 & $\mathrm{~N}(3)-\mathrm{C}(16)-\mathrm{H}(16 \mathrm{~A})$ & 108.4 \\
\hline $\mathrm{C}(12)-\mathrm{C}(11)-\mathrm{H}(11)$ & 120.1 & $\mathrm{C}(16) \# 2-\mathrm{C}(16)-\mathrm{H}(16 \mathrm{~A})$ & 108.4 \\
\hline $\mathrm{C}(7)-\mathrm{C}(12)-\mathrm{C}(11)$ & 119.2(5) & $\mathrm{N}(3)-\mathrm{C}(16)-\mathrm{H}(16 \mathrm{~B})$ & 108.4 \\
\hline$C(7)-C(12)-C(13)$ & $121.5(5)$ & $\mathrm{C}(16) \# 2-\mathrm{C}(16)-\mathrm{H}(16 \mathrm{~B})$ & 108.4 \\
\hline$C(11)-C(12)-C(13)$ & $119.2(5)$ & $\mathrm{H}(16 \mathrm{~A})-\mathrm{C}(16)-\mathrm{H}(16 \mathrm{~B})$ & 107.5 \\
\hline $\mathrm{C}(12)-\mathrm{C}(13)-\mathrm{H}(13 \mathrm{~A})$ & 109.5 & $\mathrm{~N}(3)-\mathrm{C}(17)-\mathrm{C}(18)$ & $109.6(4)$ \\
\hline $\mathrm{C}(12)-\mathrm{C}(13)-\mathrm{H}(13 \mathrm{~B})$ & 109.5 & $\mathrm{~N}(3)-\mathrm{C}(17)-\mathrm{H}(17 \mathrm{~A})$ & 109.7 \\
\hline $\mathrm{H}(13 \mathrm{~A})-\mathrm{C}(13)-\mathrm{H}(13 \mathrm{~B})$ & 109.5 & $\mathrm{C}(18)-\mathrm{C}(17)-\mathrm{H}(17 \mathrm{~A})$ & 109.7 \\
\hline $\mathrm{C}(12)-\mathrm{C}(13)-\mathrm{H}(13 \mathrm{C})$ & 109.5 & $\mathrm{~N}(3)-\mathrm{C}(17)-\mathrm{H}(17 \mathrm{~B})$ & 109.7 \\
\hline $\mathrm{H}(13 \mathrm{~A})-\mathrm{C}(13)-\mathrm{H}(13 \mathrm{C})$ & 109.5 & $\mathrm{C}(18)-\mathrm{C}(17)-\mathrm{H}(17 \mathrm{~B})$ & 109.7 \\
\hline $\mathrm{H}(13 \mathrm{~B})-\mathrm{C}(13)-\mathrm{H}(13 \mathrm{C})$ & 109.5 & $\mathrm{H}(17 \mathrm{~A})-\mathrm{C}(17)-\mathrm{H}(17 \mathrm{~B})$ & 108.2 \\
\hline $\mathrm{C}(8)-\mathrm{C}(14)-\mathrm{H}(14 \mathrm{~A})$ & 109.5 & $\mathrm{~N}(4)-\mathrm{C}(18)-\mathrm{C}(17)$ & $110.2(4)$ \\
\hline $\mathrm{C}(8)-\mathrm{C}(14)-\mathrm{H}(14 \mathrm{~B})$ & 109.5 & $\mathrm{~N}(4)-\mathrm{C}(18)-\mathrm{H}(18 \mathrm{~A})$ & 109.6 \\
\hline $\mathrm{H}(14 \mathrm{~A})-\mathrm{C}(14)-\mathrm{H}(14 \mathrm{~B})$ & 109.5 & $\mathrm{C}(17)-\mathrm{C}(18)-\mathrm{H}(18 \mathrm{~A})$ & 109.6 \\
\hline $\mathrm{C}(8)-\mathrm{C}(14)-\mathrm{H}(14 \mathrm{C})$ & 109.5 & $\mathrm{~N}(4)-\mathrm{C}(18)-\mathrm{H}(18 \mathrm{~B})$ & 109.6 \\
\hline $\mathrm{H}(14 \mathrm{~A})-\mathrm{C}(14)-\mathrm{H}(14 \mathrm{C})$ & 109.5 & $\mathrm{C}(17)-\mathrm{C}(18)-\mathrm{H}(18 \mathrm{~B})$ & 109.6 \\
\hline $\mathrm{H}(14 \mathrm{~B})-\mathrm{C}(14)-\mathrm{H}(14 \mathrm{C})$ & 109.5 & $\mathrm{H}(18 \mathrm{~A})-\mathrm{C}(18)-\mathrm{H}(18 \mathrm{~B})$ & 108.1 \\
\hline $\mathrm{C}(20) \# 2-\mathrm{C}(15)-\mathrm{N}(3)$ & $117.4(4)$ & $\mathrm{N}(4)-\mathrm{C}(19)-\mathrm{C}(20)$ & $113.1(4)$ \\
\hline $\mathrm{C}(20) \# 2-\mathrm{C}(15)-\mathrm{H}(15 \mathrm{~A})$ & 107.9 & $\mathrm{~N}(4)-\mathrm{C}(19)-\mathrm{H}(19 \mathrm{~A})$ & 109.0 \\
\hline $\mathrm{N}(3)-\mathrm{C}(15)-\mathrm{H}(15 \mathrm{~A})$ & 107.9 & $\mathrm{C}(20)-\mathrm{C}(19)-\mathrm{H}(19 \mathrm{~A})$ & 109.0 \\
\hline $\mathrm{C}(20) \# 2-\mathrm{C}(15)-\mathrm{H}(15 \mathrm{~B})$ & 107.9 & N(4)-C(19)-H(19B) & 109.0 \\
\hline $\mathrm{N}(3)-\mathrm{C}(15)-\mathrm{H}(15 \mathrm{~B})$ & 107.9 & $\mathrm{C}(20)-\mathrm{C}(19)-\mathrm{H}(19 \mathrm{~B})$ & 109.0 \\
\hline
\end{tabular}




\begin{tabular}{|c|c|c|c|}
\hline $\mathrm{H}(19 \mathrm{~A})-\mathrm{C}(19)-\mathrm{H}(19 \mathrm{~B})$ & 107.8 & $\mathrm{C}(25)-\mathrm{C}(26)-\mathrm{C}(27)$ & $119.9(5)$ \\
\hline $\mathrm{C}(15) \# 2-\mathrm{C}(20)-\mathrm{C}(19)$ & $116.5(5)$ & $\mathrm{C}(26)-\mathrm{C}(27)-\mathrm{H}(27 \mathrm{~A})$ & 109.5 \\
\hline $\mathrm{C}(15) \# 2-\mathrm{C}(20)-\mathrm{H}(20 \mathrm{~A})$ & 108.2 & $\mathrm{C}(26)-\mathrm{C}(27)-\mathrm{H}(27 \mathrm{~B})$ & 109.5 \\
\hline $\mathrm{C}(19)-\mathrm{C}(20)-\mathrm{H}(20 \mathrm{~A})$ & 108.2 & $\mathrm{H}(27 \mathrm{~A})-\mathrm{C}(27)-\mathrm{H}(27 \mathrm{~B})$ & 109.5 \\
\hline $\mathrm{C}(15) \# 2-\mathrm{C}(20)-\mathrm{H}(20 \mathrm{~B})$ & 108.2 & $\mathrm{C}(26)-\mathrm{C}(27)-\mathrm{H}(27 \mathrm{C})$ & 109.5 \\
\hline $\mathrm{C}(19)-\mathrm{C}(20)-\mathrm{H}(20 \mathrm{~B})$ & 108.2 & $\mathrm{H}(27 \mathrm{~A})-\mathrm{C}(27)-\mathrm{H}(27 \mathrm{C})$ & 109.5 \\
\hline $\mathrm{H}(20 \mathrm{~A})-\mathrm{C}(20)-\mathrm{H}(20 \mathrm{~B})$ & 107.3 & $\mathrm{H}(27 \mathrm{~B})-\mathrm{C}(27)-\mathrm{H}(27 \mathrm{C})$ & 109.5 \\
\hline $\mathrm{O}(2)-\mathrm{C}(21)-\mathrm{C}(26)$ & $121.0(4)$ & $\mathrm{C}(22)-\mathrm{C}(28)-\mathrm{H}(28 \mathrm{~A})$ & 109.5 \\
\hline $\mathrm{O}(2)-\mathrm{C}(21)-\mathrm{C}(22)$ & $118.2(4)$ & $\mathrm{C}(22)-\mathrm{C}(28)-\mathrm{H}(28 \mathrm{~B})$ & 109.5 \\
\hline$C(26)-C(21)-C(22)$ & $120.8(4)$ & $\mathrm{H}(28 \mathrm{~A})-\mathrm{C}(28)-\mathrm{H}(28 \mathrm{~B})$ & 109.5 \\
\hline$C(23)-C(22)-C(21)$ & $116.5(4)$ & $\mathrm{C}(22)-\mathrm{C}(28)-\mathrm{H}(28 \mathrm{C})$ & 109.5 \\
\hline$C(23)-C(22)-C(28)$ & $123.6(5)$ & $\mathrm{H}(28 \mathrm{~A})-\mathrm{C}(28)-\mathrm{H}(28 \mathrm{C})$ & 109.5 \\
\hline $\mathrm{C}(21)-\mathrm{C}(22)-\mathrm{C}(28)$ & $119.9(5)$ & $\mathrm{H}(28 \mathrm{~B})-\mathrm{C}(28)-\mathrm{H}(28 \mathrm{C})$ & 109.5 \\
\hline$C(24)-C(23)-C(22)$ & $124.2(5)$ & $\mathrm{C}(2)-\mathrm{N}(1)-\mathrm{C}(3)$ & $110.8(4)$ \\
\hline $\mathrm{C}(24)-\mathrm{C}(23)-\mathrm{H}(23)$ & 117.9 & $\mathrm{C}(2)-\mathrm{N}(1)-\mathrm{C}(1)$ & $110.6(4)$ \\
\hline $\mathrm{C}(22)-\mathrm{C}(23)-\mathrm{H}(23)$ & 117.9 & $\mathrm{C}(3)-\mathrm{N}(1)-\mathrm{C}(1)$ & $108.9(4)$ \\
\hline$C(23)-C(24)-C(25)$ & $118.8(5)$ & $\mathrm{C}(2)-\mathrm{N}(1)-\mathrm{Zr}(1)$ & $110.6(3)$ \\
\hline $\mathrm{C}(23)-\mathrm{C}(24)-\mathrm{H}(24)$ & 120.6 & $\mathrm{C}(3)-\mathrm{N}(1)-\mathrm{Zr}(1)$ & $104.4(3)$ \\
\hline $\mathrm{C}(25)-\mathrm{C}(24)-\mathrm{H}(24)$ & 120.6 & $\mathrm{C}(1)-\mathrm{N}(1)-\mathrm{Zr}(1)$ & $111.4(3)$ \\
\hline$C(26)-C(25)-C(24)$ & 120.4(4) & $\mathrm{C}(5)-\mathrm{N}(2)-\mathrm{C}(4)$ & $113.7(5)$ \\
\hline $\mathrm{C}(26)-\mathrm{C}(25)-\mathrm{H}(25)$ & 119.8 & $\mathrm{C}(5)-\mathrm{N}(2)-\mathrm{Zr}(1)$ & $117.8(3)$ \\
\hline $\mathrm{C}(24)-\mathrm{C}(25)-\mathrm{H}(25)$ & 119.8 & $\mathrm{C}(4)-\mathrm{N}(2)-\mathrm{Zr}(1)$ & $121.3(3)$ \\
\hline $\mathrm{C}(21)-\mathrm{C}(26)-\mathrm{C}(25)$ & 119.1(4) & $\mathrm{C}(17)-\mathrm{N}(3)-\mathrm{C}(16)$ & $112.3(4)$ \\
\hline$C(21)-C(26)-C(27)$ & $120.9(4)$ & C(17)-N(3)-C(15) & 109.1(4) \\
\hline
\end{tabular}




\begin{tabular}{|c|c|c|c|}
\hline $\mathrm{C}(16)-\mathrm{N}(3)-\mathrm{C}(15)$ & 106.1(4) & $\mathrm{O}(1) \# 1-\mathrm{Zr}(1)-\mathrm{N}(1)$ & 92.91(14) \\
\hline $\mathrm{C}(17)-\mathrm{N}(3)-\operatorname{Zr}(2)$ & 105.2(3) & $\mathrm{N}(2)-\mathrm{Zr}(1)-\mathrm{N}(1)$ & $74.41(17)$ \\
\hline $\mathrm{C}(16)-\mathrm{N}(3)-\mathrm{Zr}(2)$ & $111.6(3)$ & $\mathrm{N}(2) \# 1-\mathrm{Zr}(1)-\mathrm{N}(1)$ & $78.32(16)$ \\
\hline $\mathrm{C}(15)-\mathrm{N}(3)-\mathrm{Zr}(2)$ & $112.7(3)$ & $\mathrm{N}(1) \# 1-\mathrm{Zr}(1)-\mathrm{N}(1)$ & $76.6(2)$ \\
\hline $\mathrm{C}(19)-\mathrm{N}(4)-\mathrm{C}(18)$ & $111.8(4)$ & $\mathrm{O}(2) \# 2-\mathrm{Zr}(2)-\mathrm{O}(2)$ & $100.6(2)$ \\
\hline $\mathrm{C}(19)-\mathrm{N}(4)-\mathrm{Zr}(2)$ & $120.5(3)$ & $\mathrm{O}(2) \# 2-\mathrm{Zr}(2)-\mathrm{N}(4)$ & $103.36(14)$ \\
\hline $\mathrm{C}(18)-\mathrm{N}(4)-\mathrm{Zr}(2)$ & $121.0(3)$ & $\mathrm{O}(2)-\mathrm{Zr}(2)-\mathrm{N}(4)$ & $98.64(15)$ \\
\hline $\mathrm{C}(7)-\mathrm{O}(1)-\mathrm{Zr}(1)$ & $164.2(3)$ & $\mathrm{O}(2) \# 2-\mathrm{Zr}(2)-\mathrm{N}(4) \# 2$ & $98.64(15)$ \\
\hline $\mathrm{C}(21)-\mathrm{O}(2)-\mathrm{Zr}(2)$ & $163.8(3)$ & $\mathrm{O}(2)-\mathrm{Zr}(2)-\mathrm{N}(4) \# 2$ & $103.36(14)$ \\
\hline $\mathrm{O}(1)-\operatorname{Zr}(1)-\mathrm{O}(1) \# 1$ & $98.4(2)$ & $\mathrm{N}(4)-\mathrm{Zr}(2)-\mathrm{N}(4) \# 2$ & 145.3(3) \\
\hline $\mathrm{O}(1)-\mathrm{Zr}(1)-\mathrm{N}(2)$ & $96.42(15)$ & $\mathrm{O}(2) \# 2-\mathrm{Zr}(2)-\mathrm{N}(3)$ & $91.72(14)$ \\
\hline $\mathrm{O}(1) \# 1-\mathrm{Zr}(1)-\mathrm{N}(2)$ & $106.28(14)$ & $\mathrm{O}(2)-\mathrm{Zr}(2)-\mathrm{N}(3)$ & $166.89(16)$ \\
\hline $\mathrm{O}(1)-\mathrm{Zr}(1)-\mathrm{N}(2) \# 1$ & $106.28(14)$ & $\mathrm{N}(4)-\mathrm{Zr}(2)-\mathrm{N}(3)$ & $73.90(16)$ \\
\hline $\mathrm{O}(1) \# 1-\mathrm{Zr}(1)-\mathrm{N}(2) \# 1$ & $96.42(15)$ & $\mathrm{N}(4) \# 2-\mathrm{Zr}(2)-\mathrm{N}(3)$ & $78.95(16)$ \\
\hline $\mathrm{N}(2)-\operatorname{Zr}(1)-\mathrm{N}(2) \# 1$ & $145.1(3)$ & $\mathrm{O}(2) \# 2-\mathrm{Zr}(2)-\mathrm{N}(3) \# 2$ & $166.89(16)$ \\
\hline $\mathrm{O}(1)-\operatorname{Zr}(1)-\mathrm{N}(1) \# 1$ & 92.91(14) & $\mathrm{O}(2)-\mathrm{Zr}(2)-\mathrm{N}(3) \# 2$ & $91.72(14)$ \\
\hline $\mathrm{O}(1) \# 1-\mathrm{Zr}(1)-\mathrm{N}(1) \# 1$ & $167.12(16)$ & $\mathrm{N}(4)-\mathrm{Zr}(2)-\mathrm{N}(3) \# 2$ & $78.95(16)$ \\
\hline $\mathrm{N}(2)-\operatorname{Zr}(1)-\mathrm{N}(1) \# 1$ & $78.32(16)$ & $\mathrm{N}(4) \# 2-\mathrm{Zr}(2)-\mathrm{N}(3) \# 2$ & $73.90(16)$ \\
\hline $\mathrm{N}(2) \# 1-\mathrm{Zr}(1)-\mathrm{N}(1) \# 1$ & $74.41(17)$ & $\mathrm{N}(3)-\operatorname{Zr}(2)-\mathrm{N}(3) \# 2$ & $76.4(2)$ \\
\hline $\mathrm{O}(1)-\mathrm{Zr}(1)-\mathrm{N}(1)$ & $167.12(16)$ & & \\
\hline
\end{tabular}

Symmetry transformations used to generate equivalent atoms: $\# 1-x+2,-y, z \# 2-x+1,-y+1, z$ 
Table S3. Anisotropic displacement parameters $\left(\AA^{2} \times 10^{3}\right)$ for $(\mathrm{CBC}) \mathrm{Zr}\left(\mathrm{O}-2,6-\mathrm{C}_{6} \mathrm{H}_{3} \mathrm{Me}_{2}\right)_{2}, \mathbf{5}$.

$\begin{array}{llllll}\mathrm{U}_{11} & \mathrm{U}_{22} & \mathrm{U}_{33} & \mathrm{U}_{23} & \mathrm{U}_{13} & \mathrm{U}_{12}\end{array}$

\begin{tabular}{|c|c|c|c|c|c|c|}
\hline$C(1)$ & $20(2)$ & $38(3)$ & $30(2)$ & $-11(2)$ & $-18(2)$ & $14(2)$ \\
\hline $\mathrm{C}(2)$ & 41(3) & $45(3)$ & $6(2)$ & $-2(2)$ & $-3(2)$ & $0(2)$ \\
\hline $\mathrm{C}(3)$ & $23(2)$ & $42(3)$ & $17(2)$ & $-11(2)$ & $-4(2)$ & $0(2)$ \\
\hline$C(4)$ & $32(2)$ & $39(3)$ & $17(2)$ & $-6(2)$ & $5(2)$ & $-4(2)$ \\
\hline $\mathrm{C}(5)$ & $42(3)$ & $33(3)$ & $29(2)$ & $9(2)$ & $15(2)$ & $-1(2)$ \\
\hline$C(6)$ & $45(2)$ & $28(2)$ & $34(2)$ & $9(2)$ & $17(2)$ & $14(2)$ \\
\hline $\mathrm{C}(7)$ & $18(2)$ & $26(2)$ & $21(2)$ & $-3(2)$ & $0(2)$ & $5(2)$ \\
\hline $\mathrm{C}(8)$ & $25(2)$ & $18(2)$ & $18(3)$ & $-10(2)$ & $-4(2)$ & $7(2)$ \\
\hline $\mathrm{C}(9)$ & $31(2)$ & $31(2)$ & $27(3)$ & $-17(2)$ & $-23(2)$ & $9(2)$ \\
\hline$C(10)$ & $48(3)$ & $23(2)$ & $15(2)$ & $-2(2)$ & $-11(2)$ & $10(2)$ \\
\hline $\mathrm{C}(11)$ & $45(3)$ & $25(2)$ & $10(2)$ & $-1(2)$ & $-1(2)$ & $0(2)$ \\
\hline $\mathrm{C}(12)$ & $28(2)$ & $44(3)$ & $10(2)$ & $1(2)$ & $-3(2)$ & $-1(2)$ \\
\hline $\mathrm{C}(13)$ & $33(3)$ & $92(6)$ & $21(3)$ & 4(4) & $4(2)$ & $-23(3)$ \\
\hline$C(14)$ & $38(3)$ & $32(3)$ & $46(5)$ & $-13(2)$ & $-9(3)$ & $2(2)$ \\
\hline $\mathrm{C}(15)$ & $47(3)$ & $39(3)$ & $17(2)$ & $-7(2)$ & $-5(2)$ & $24(2)$ \\
\hline$C(16)$ & $27(3)$ & $28(3)$ & 19(3) & $-5(2)$ & $-2(2)$ & $13(2)$ \\
\hline $\mathrm{C}(17)$ & $51(3)$ & $24(2)$ & $23(2)$ & $-5(2)$ & $-7(2)$ & $-1(2)$ \\
\hline $\mathrm{C}(18)$ & $42(3)$ & $40(3)$ & $26(2)$ & 2(2) & $-7(2)$ & $-6(2)$ \\
\hline$C(19)$ & $34(2)$ & $53(3)$ & $19(2)$ & $15(3)$ & $-1(2)$ & $-3(2)$ \\
\hline
\end{tabular}




\begin{tabular}{|c|c|c|c|c|c|c|}
\hline$C(20)$ & $41(2)$ & $48(3)$ & $27(2)$ & $12(2)$ & $5(2)$ & $19(2)$ \\
\hline $\mathrm{C}(21)$ & $30(2)$ & $27(3)$ & $5(2)$ & $-5(2)$ & $1(2)$ & $8(2)$ \\
\hline$C(22)$ & $31(2)$ & $28(3)$ & $14(2)$ & $2(2)$ & $-3(2)$ & $-1(2)$ \\
\hline$C(23)$ & $29(2)$ & $40(3)$ & $13(2)$ & $1(2)$ & $2(2)$ & $2(2)$ \\
\hline$C(24)$ & $33(2)$ & $41(3)$ & $9(2)$ & $-7(2)$ & $-10(2)$ & $9(2)$ \\
\hline$C(25)$ & $28(2)$ & $31(2)$ & $18(2)$ & $1(2)$ & $-15(2)$ & $3(2)$ \\
\hline$C(26)$ & $20(2)$ & $27(2)$ & $15(2)$ & $1(2)$ & $0(2)$ & $1(2)$ \\
\hline$C(27)$ & $30(3)$ & $20(3)$ & $20(3)$ & $4(2)$ & $1(2)$ & $-14(2)$ \\
\hline$C(28)$ & $78(4)$ & $41(4)$ & $17(3)$ & $3(2)$ & $12(3)$ & $-9(3)$ \\
\hline $\mathrm{N}(1)$ & $23(2)$ & $42(2)$ & $20(2)$ & $-9(2)$ & $-6(2)$ & $8(2)$ \\
\hline $\mathrm{N}(2)$ & $28(2)$ & $37(2)$ & $19(2)$ & $-2(2)$ & $9(1)$ & $6(1)$ \\
\hline $\mathrm{N}(3)$ & $31(2)$ & $26(2)$ & $11(2)$ & $-4(2)$ & $-1(1)$ & $11(1)$ \\
\hline $\mathrm{N}(4)$ & $38(2)$ & $27(2)$ & $14(2)$ & $3(1)$ & $2(2)$ & $3(1)$ \\
\hline $\mathrm{O}(1)$ & $17(1)$ & $50(2)$ & $15(2)$ & $6(2)$ & $-3(1)$ & $7(1)$ \\
\hline $\mathrm{O}(2)$ & $45(2)$ & $27(2)$ & $14(2)$ & $1(2)$ & $11(2)$ & $3(1)$ \\
\hline $\operatorname{Zr}(1)$ & $7(1)$ & $44(1)$ & $9(1)$ & 0 & 0 & $5(1)$ \\
\hline $\operatorname{Zr}(2)$ & $28(1)$ & $23(1)$ & 11(1) & 0 & 0 & $6(1)$ \\
\hline
\end{tabular}

The anisotropic displacement factor exponent takes the form:

$-2 \pi^{2}\left[\mathrm{~h}^{2} \mathrm{a}^{*} \mathrm{U}_{11}+\ldots+2 \mathrm{~h} \mathrm{k} \mathrm{a}^{*} \mathrm{~b} * \mathrm{U}_{12}\right]$ 
Table S4. Hydrogen coordinates $\left(\begin{array}{ll}x & \left.10^{4}\right)\end{array}\right)$ and isotropic displacement parameters $\left(\AA^{2} \times 10^{3}\right)$ for $(\mathrm{CBC}) \mathrm{Zr}\left(\mathrm{O}-2,6-\mathrm{C}_{6} \mathrm{H}_{3} \mathrm{Me}_{2}\right)_{2}, \mathbf{5}$.

\begin{tabular}{|c|c|c|c|c|}
\hline & $\mathrm{x}$ & $\mathrm{y}$ & $\mathrm{z}$ & $\mathrm{U}(\mathrm{eq})$ \\
\hline $\mathrm{H}(1 \mathrm{~A})$ & 12295 & -1604 & 5642 & 35 \\
\hline $\mathrm{H}(1 \mathrm{~B})$ & 12180 & -1508 & 4827 & 35 \\
\hline $\mathrm{H}(2 \mathrm{~A})$ & 10257 & -1143 & 6107 & 37 \\
\hline $\mathrm{H}(2 \mathrm{~B})$ & 11006 & -121 & 6374 & 37 \\
\hline $\mathrm{H}(3 \mathrm{~A})$ & 12569 & 374 & 4951 & 33 \\
\hline $\mathrm{H}(3 \mathrm{~B})$ & 12465 & 496 & 5769 & 33 \\
\hline $\mathrm{H}(4 \mathrm{~A})$ & 11174 & 1901 & 5684 & 35 \\
\hline $\mathrm{H}(4 \mathrm{~B})$ & 12089 & 2204 & 5107 & 35 \\
\hline $\mathrm{H}(5 \mathrm{~A})$ & 9500 & 2555 & 4242 & 42 \\
\hline $\mathrm{H}(5 \mathrm{~B})$ & 10340 & 3236 & 4727 & 42 \\
\hline $\mathrm{H}(6 \mathrm{~A})$ & 8527 & 3240 & 5193 & 43 \\
\hline $\mathrm{H}(6 \mathrm{~B})$ & 9381 & 2634 & 5707 & 43 \\
\hline $\mathrm{H}(9)$ & 6013 & 1000 & 2330 & 35 \\
\hline $\mathrm{H}(10)$ & 6906 & 2272 & 1646 & 35 \\
\hline $\mathrm{H}(11)$ & 8810 & 2685 & 1831 & 32 \\
\hline $\mathrm{H}(13 \mathrm{~A})$ & 10343 & 2172 & 3283 & 73 \\
\hline $\mathrm{H}(13 \mathrm{~B})$ & 10428 & 2581 & 2500 & 73 \\
\hline $\mathrm{H}(13 \mathrm{C})$ & 10630 & 1289 & 2687 & 73 \\
\hline $\mathrm{H}(14 \mathrm{~A})$ & 6472 & 386 & 3902 & 58 \\
\hline
\end{tabular}




\begin{tabular}{|c|c|c|c|c|}
\hline $\mathrm{H}(14 \mathrm{~B})$ & 7217 & -637 & 3625 & 58 \\
\hline $\mathrm{H}(14 \mathrm{C})$ & 6046 & -347 & 3263 & 58 \\
\hline $\mathrm{H}(15 \mathrm{~A})$ & 6852 & 3045 & 4138 & 41 \\
\hline $\mathrm{H}(15 \mathrm{~B})$ & 6920 & 3028 & 3319 & 41 \\
\hline $\mathrm{H}(16 \mathrm{~A})$ & 5258 & 3983 & 2655 & 30 \\
\hline $\mathrm{H}(16 \mathrm{~B})$ & 6187 & 4852 & 2913 & 30 \\
\hline $\mathrm{H}(17 \mathrm{~A})$ & 5056 & 2397 & 4082 & 39 \\
\hline $\mathrm{H}(17 \mathrm{~B})$ & 4896 & 2472 & 3263 & 39 \\
\hline $\mathrm{H}(18 \mathrm{~A})$ & 3317 & 3543 & 3360 & 43 \\
\hline $\mathrm{H}(18 \mathrm{~B})$ & 3166 & 2606 & 3946 & 43 \\
\hline $\mathrm{H}(19 \mathrm{~A})$ & 2356 & 5069 & 4775 & 43 \\
\hline $\mathrm{H}(19 \mathrm{~B})$ & 1878 & 4130 & 4265 & 43 \\
\hline $\mathrm{H}(20 \mathrm{~A})$ & 2365 & 5203 & 3293 & 46 \\
\hline $\mathrm{H}(20 \mathrm{~B})$ & 1621 & 5966 & 3790 & 46 \\
\hline $\mathrm{H}(23)$ & 2326 & 6388 & 7210 & 33 \\
\hline $\mathrm{H}(24)$ & 2892 & 8183 & 7422 & 33 \\
\hline $\mathrm{H}(25)$ & 4172 & 9054 & 6667 & 31 \\
\hline $\mathrm{H}(27 \mathrm{~A})$ & 5670 & 7781 & 5334 & 35 \\
\hline $\mathrm{H}(27 \mathrm{~B})$ & 5489 & 8939 & 5737 & 35 \\
\hline $\mathrm{H}(27 \mathrm{C})$ & 4671 & 8605 & 5117 & 35 \\
\hline $\mathrm{H}(28 \mathrm{~A})$ & 2637 & 4567 & 6684 & 68 \\
\hline $\mathrm{H}(28 \mathrm{~B})$ & 3606 & 4455 & 6118 & 68 \\
\hline $\mathrm{H}(28 \mathrm{C})$ & 2370 & 4826 & 5892 & 68 \\
\hline
\end{tabular}


Figure S2. ${ }^{1} \mathrm{H}$ NMR of $(\mathrm{CBC}) \mathrm{Zr}\left(\mathrm{O}-2,6-\mathrm{C}_{6} \mathrm{H}_{3} \mathrm{Me}_{2}\right)_{2}, \mathbf{5}$.

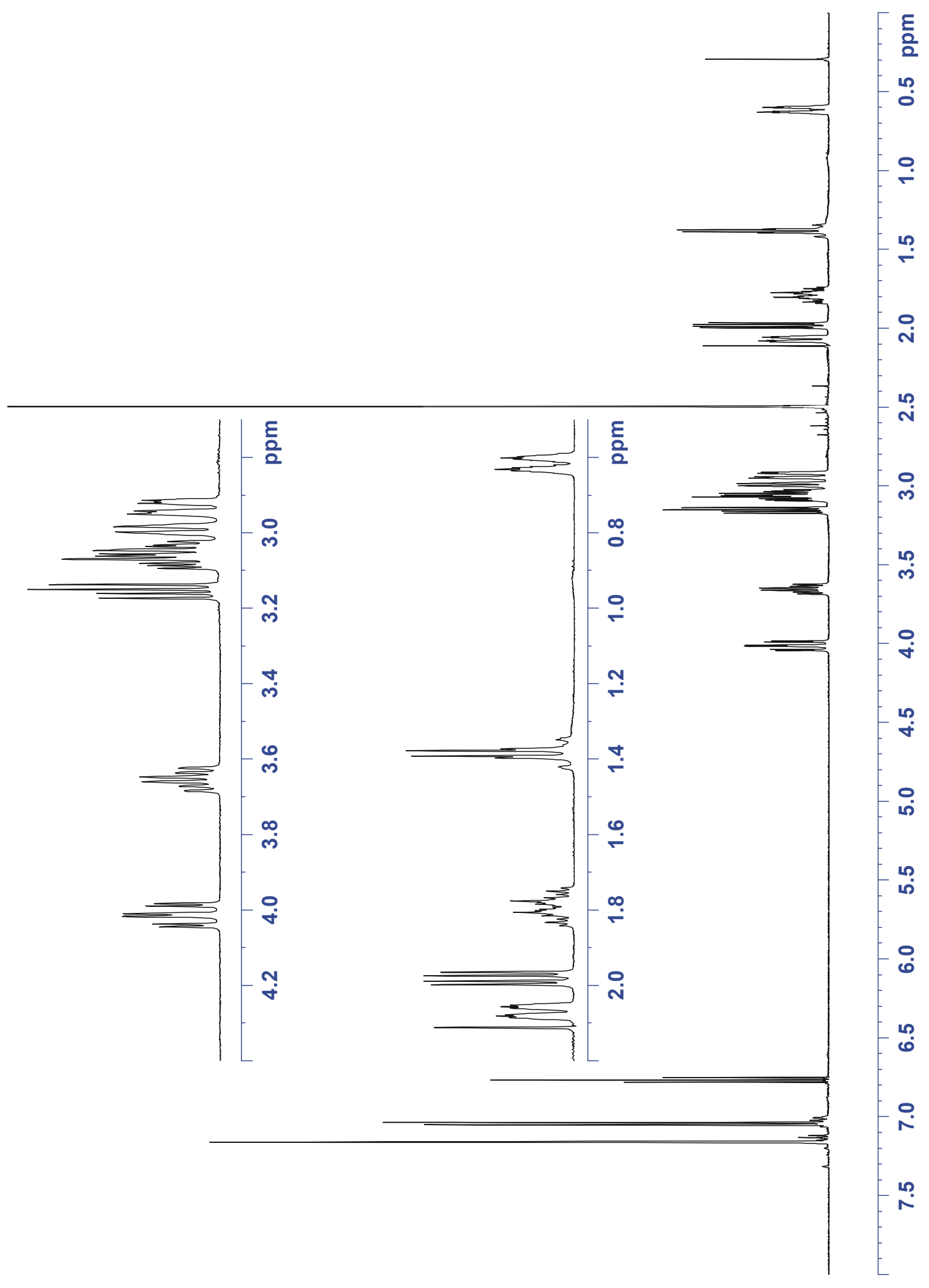


Figure S3. ORTEP Drawing of $(\mathrm{CBC}) \mathrm{Zr}\left(\mathrm{CH}_{2} \mathrm{Si}\left(\mathrm{CH}_{3}\right)_{3}\right)_{2}, \mathbf{9}$.

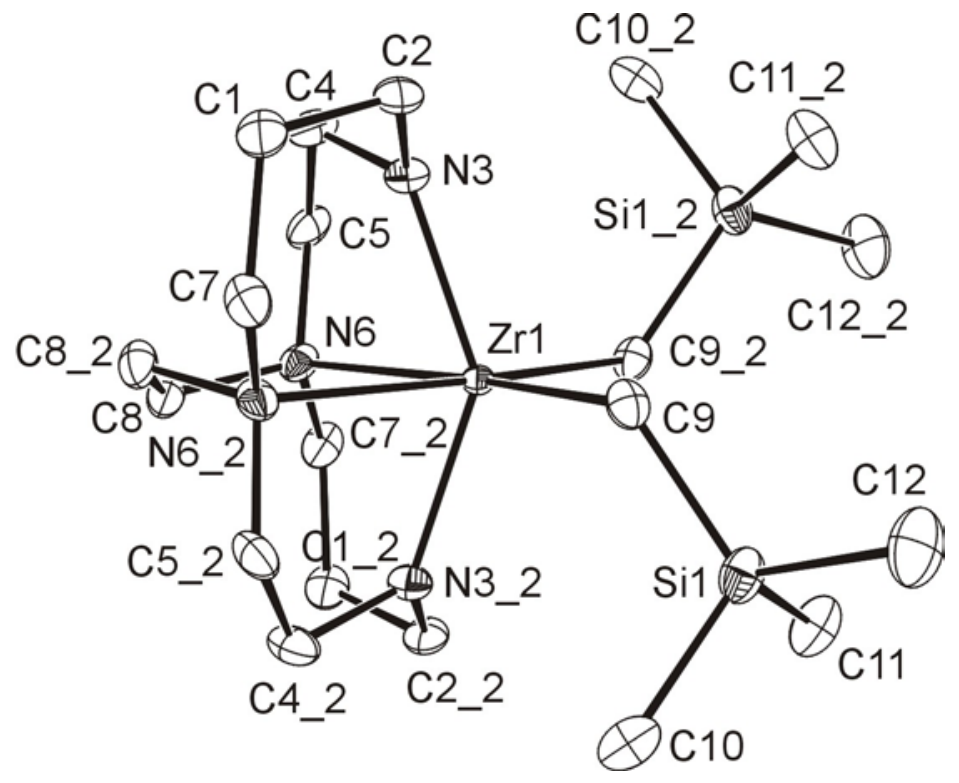


Table S5. Atomic coordinates (x $\left.10^{4}\right)$ and equivalent isotropic displacement parameters $\left(\AA^{2} \times 10^{3}\right)$ for $(\mathrm{CBC}) \mathrm{Zr}\left(\mathrm{CH}_{2} \mathrm{Si}\left(\mathrm{CH}_{3}\right)_{3}\right)_{2}, 9$.

$\begin{array}{lcccc} & \mathrm{x} & \mathrm{y} & \mathrm{z} & \mathrm{U}(\mathrm{eq}) \\ \mathrm{C}(1) & 8061(2) & 1236(1) & 5174(3) & 22(1) \\ \mathrm{C}(2) & 8629(2) & 1850(1) & 4694(3) & 20(1) \\ \mathrm{C}(4) & 10107(2) & 1268(1) & 4757(3) & 22(1) \\ \mathrm{C}(5) & 11039(2) & 1165(1) & 5736(3) & 21(1) \\ \mathrm{C}(7) & 11904(2) & 1190(1) & 8199(3) & 20(1) \\ \mathrm{C}(8) & 10524(2) & 462(1) & 7647(3) & 21(1) \\ \mathrm{C}(9) & 8871(2) & 2994(1) & 7772(3) & 18(1) \\ \mathrm{C}(10) & 8731(2) & 3008(2) & 10995(3) & 27(1) \\ \mathrm{C}(11) & 10147(2) & 3950(2) & 10042(3) & 28(1) \\ \mathrm{C}(12) & 8150(2) & 4335(2) & 9107(4) & 34(1) \\ \mathrm{N}(3) & 9603(1) & 1826(1) & 5359(2) & 16(1) \\ \mathrm{N}(6) & 10962(1) & 1139(1) & 7275(2) & 17(1) \\ \mathrm{Si}(1) & 8975(1) & 3550(1) & 9435(1) & 20(1) \\ \mathrm{Zr}(1) & 10000 & 2194(1) & 7500 & 10(1)\end{array}$

$\mathrm{U}(\mathrm{eq})$ is defined as one third of the trace of the orthogonalized $\mathrm{U}_{\mathrm{ij}}$ tensor. 


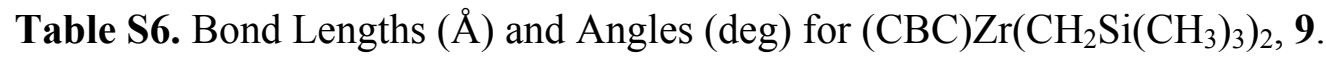

\begin{tabular}{|c|c|c|c|}
\hline \multicolumn{2}{|c|}{ Bond Lengths } & $\mathrm{C}(8)-\mathrm{H}(8 \mathrm{~B})$ & 0.9900 \\
\hline$C(1)-C(7) \# 1$ & $1.526(4)$ & $\mathrm{C}(9)-\mathrm{Si}(1)$ & $1.855(3)$ \\
\hline$C(1)-C(2)$ & $1.534(4)$ & $\mathrm{C}(9)-\operatorname{Zr}(1)$ & $2.290(2)$ \\
\hline $\mathrm{C}(1)-\mathrm{H}(1 \mathrm{~A})$ & 0.9900 & $\mathrm{C}(9)-\mathrm{H}(9 \mathrm{~A})$ & 0.9900 \\
\hline $\mathrm{C}(1)-\mathrm{H}(1 \mathrm{~B})$ & 0.9900 & $\mathrm{C}(9)-\mathrm{H}(9 \mathrm{~B})$ & 0.9900 \\
\hline$C(2)-N(3)$ & $1.464(3)$ & $\mathrm{C}(10)-\mathrm{Si}(1)$ & $1.872(3)$ \\
\hline $\mathrm{C}(2)-\mathrm{H}(2 \mathrm{~A})$ & 0.9900 & $\mathrm{C}(10)-\mathrm{H}(10 \mathrm{~A})$ & 0.9800 \\
\hline $\mathrm{C}(2)-\mathrm{H}(2 \mathrm{~B})$ & 0.9900 & $\mathrm{C}(10)-\mathrm{H}(10 \mathrm{~B})$ & 0.9800 \\
\hline $\mathrm{C}(4)-\mathrm{N}(3)$ & $1.452(3)$ & $\mathrm{C}(10)-\mathrm{H}(10 \mathrm{C})$ & 0.9800 \\
\hline$C(4)-C(5)$ & $1.521(4)$ & $\mathrm{C}(11)-\mathrm{Si}(1)$ & $1.878(3)$ \\
\hline $\mathrm{C}(4)-\mathrm{H}(4 \mathrm{~A})$ & 0.9900 & $\mathrm{C}(11)-\mathrm{H}(11 \mathrm{~A})$ & 0.9800 \\
\hline $\mathrm{C}(4)-\mathrm{H}(4 \mathrm{~B})$ & 0.9900 & $\mathrm{C}(11)-\mathrm{H}(11 \mathrm{~B})$ & 0.9800 \\
\hline $\mathrm{C}(5)-\mathrm{N}(6)$ & $1.479(3)$ & $\mathrm{C}(11)-\mathrm{H}(11 \mathrm{C})$ & 0.9800 \\
\hline $\mathrm{C}(5)-\mathrm{H}(5 \mathrm{~A})$ & 0.9900 & $\mathrm{C}(12)-\mathrm{Si}(1)$ & $1.885(3)$ \\
\hline $\mathrm{C}(5)-\mathrm{H}(5 \mathrm{~B})$ & 0.9900 & $\mathrm{C}(12)-\mathrm{H}(12 \mathrm{~A})$ & 0.9800 \\
\hline $\mathrm{C}(7)-\mathrm{N}(6)$ & $1.502(3)$ & $\mathrm{C}(12)-\mathrm{H}(12 \mathrm{~B})$ & 0.9800 \\
\hline$C(7)-C(1) \# 1$ & $1.526(4)$ & $\mathrm{C}(12)-\mathrm{H}(12 \mathrm{C})$ & 0.9800 \\
\hline $\mathrm{C}(7)-\mathrm{H}(7 \mathrm{~A})$ & 0.9900 & $\mathrm{~N}(3)-\operatorname{Zr}(1)$ & $2.103(2)$ \\
\hline $\mathrm{C}(7)-\mathrm{H}(7 \mathrm{~B})$ & 0.9900 & $\mathrm{~N}(6)-\operatorname{Zr}(1)$ & $2.452(2)$ \\
\hline $\mathrm{C}(8)-\mathrm{N}(6)$ & $1.483(3)$ & $\mathrm{Zr}(1)-\mathrm{N}(3) \# 1$ & $2.103(2)$ \\
\hline $\mathrm{C}(8)-\mathrm{C}(8) \# 1$ & $1.531(5)$ & $\operatorname{Zr}(1)-C(9) \# 1$ & $2.290(2)$ \\
\hline $\mathrm{C}(8)-\mathrm{H}(8 \mathrm{~A})$ & 0.9900 & $\operatorname{Zr}(1)-\mathrm{N}(6) \# 1$ & $2.452(2)$ \\
\hline
\end{tabular}


Bond Angles

C(7)\#1-C(1)-C(2) 115.1(2)

C(7)\#1-C(1)-H(1A) 108.5

$\mathrm{C}(2)-\mathrm{C}(1)-\mathrm{H}(1 \mathrm{~A}) \quad 108.5$

C(7)\#1-C(1)-H(1B) 108.5

$\mathrm{C}(2)-\mathrm{C}(1)-\mathrm{H}(1 \mathrm{~B}) \quad 108.5$

H(1A)-C(1)-H(1B) $\quad 107.5$

$\mathrm{N}(3)-\mathrm{C}(2)-\mathrm{C}(1) \quad 114.1(2)$

$\mathrm{N}(3)-\mathrm{C}(2)-\mathrm{H}(2 \mathrm{~A}) \quad 108.7$

$\mathrm{C}(1)-\mathrm{C}(2)-\mathrm{H}(2 \mathrm{~A}) \quad 108.7$

$\mathrm{N}(3)-\mathrm{C}(2)-\mathrm{H}(2 \mathrm{~B}) \quad 108.7$

$\mathrm{C}(1)-\mathrm{C}(2)-\mathrm{H}(2 \mathrm{~B}) \quad 108.7$

$\mathrm{H}(2 \mathrm{~A})-\mathrm{C}(2)-\mathrm{H}(2 \mathrm{~B}) \quad 107.6$

$\mathrm{N}(3)-\mathrm{C}(4)-\mathrm{C}(5) \quad 109.4(2)$

$\mathrm{N}(3)-\mathrm{C}(4)-\mathrm{H}(4 \mathrm{~A}) \quad 109.8$

$\mathrm{C}(5)-\mathrm{C}(4)-\mathrm{H}(4 \mathrm{~A}) \quad 109.8$

$\mathrm{N}(3)-\mathrm{C}(4)-\mathrm{H}(4 \mathrm{~B}) \quad 109.8$

$\mathrm{C}(5)-\mathrm{C}(4)-\mathrm{H}(4 \mathrm{~B}) \quad 109.8$

H(4A)-C(4)-H(4B) 108.2

$\mathrm{N}(6)-\mathrm{C}(5)-\mathrm{C}(4)$

$\mathrm{N}(6)-\mathrm{C}(5)-\mathrm{H}(5 \mathrm{~A}) \quad 109.4$

C(4)-C(5)-H(5A) 109.4

$\mathrm{N}(6)-\mathrm{C}(5)-\mathrm{H}(5 \mathrm{~B}) \quad 109.4$

$\mathrm{C}(4)-\mathrm{C}(5)-\mathrm{H}(5 \mathrm{~B})$
$111.3(2)$

109.4
H(5A)-C(5)-H(5B) 108.0

$\mathrm{N}(6)-\mathrm{C}(7)-\mathrm{C}(1) \# 1 \quad 115.5(2)$

$\mathrm{N}(6)-\mathrm{C}(7)-\mathrm{H}(7 \mathrm{~A}) \quad 108.4$

C(1)\#1-C(7)-H(7A) 108.4

$\mathrm{N}(6)-\mathrm{C}(7)-\mathrm{H}(7 \mathrm{~B}) \quad 108.4$

C(1)\#1-C(7)-H(7B) 108.4

H(7A)-C(7)-H(7B) 107.5

N(6)-C(8)-C(8)\#1 115.91(14)

$\mathrm{N}(6)-\mathrm{C}(8)-\mathrm{H}(8 \mathrm{~A}) \quad 108.3$

C(8)\#1-C(8)-H(8A) 108.3

$\mathrm{N}(6)-\mathrm{C}(8)-\mathrm{H}(8 \mathrm{~B}) \quad 108.3$

C(8)\#1-C(8)-H(8B) 108.3

$\mathrm{H}(8 \mathrm{~A})-\mathrm{C}(8)-\mathrm{H}(8 \mathrm{~B}) \quad 107.4$

$\operatorname{Si}(1)-C(9)-\operatorname{Zr}(1) \quad 120.69(12)$

$\mathrm{Si}(1)-\mathrm{C}(9)-\mathrm{H}(9 \mathrm{~A}) \quad 107.2$

$\operatorname{Zr}(1)-C(9)-H(9 A) \quad 107.2$

$\mathrm{Si}(1)-\mathrm{C}(9)-\mathrm{H}(9 \mathrm{~B}) \quad 107.2$

$\mathrm{Zr}(1)-\mathrm{C}(9)-\mathrm{H}(9 \mathrm{~B}) \quad 107.2$

H(9A)-C(9)-H(9B) 106.8

$\mathrm{Si}(1)-\mathrm{C}(10)-\mathrm{H}(10 \mathrm{~A}) \quad 109.5$

$\mathrm{Si}(1)-\mathrm{C}(10)-\mathrm{H}(10 \mathrm{~B}) \quad 109.5$

H(10A)-C(10)-H(10B) 109.5

Si(1)-C(10)-H(10C) $\quad 109.5$

H(10A)-C(10)-H(10C) 109.5 


\begin{tabular}{|c|c|c|c|}
\hline $\mathrm{H}(10 \mathrm{~B})-\mathrm{C}(10)-\mathrm{H}(10 \mathrm{C})$ & 109.5 & $C(9)-\operatorname{Si}(1)-C(10)$ & $111.80(12)$ \\
\hline $\mathrm{Si}(1)-\mathrm{C}(11)-\mathrm{H}(11 \mathrm{~A})$ & 109.5 & $\mathrm{C}(9)-\mathrm{Si}(1)-\mathrm{C}(11)$ & $113.18(13)$ \\
\hline $\mathrm{Si}(1)-\mathrm{C}(11)-\mathrm{H}(11 \mathrm{~B})$ & 109.5 & $C(10)-\operatorname{Si}(1)-C(11)$ & $106.35(14)$ \\
\hline $\mathrm{H}(11 \mathrm{~A})-\mathrm{C}(11)-\mathrm{H}(11 \mathrm{~B})$ & 109.5 & $\mathrm{C}(9)-\mathrm{Si}(1)-\mathrm{C}(12)$ & $110.02(13)$ \\
\hline $\mathrm{Si}(1)-\mathrm{C}(11)-\mathrm{H}(11 \mathrm{C})$ & 109.5 & $C(10)-\operatorname{Si}(1)-C(12)$ & $108.65(14)$ \\
\hline $\mathrm{H}(11 \mathrm{~A})-\mathrm{C}(11)-\mathrm{H}(11 \mathrm{C})$ & 109.5 & $C(11)-\operatorname{Si}(1)-C(12)$ & $106.57(13)$ \\
\hline $\mathrm{H}(11 \mathrm{~B})-\mathrm{C}(11)-\mathrm{H}(11 \mathrm{C})$ & 109.5 & $\mathrm{~N}(3) \# 1-\mathrm{Zr}(1)-\mathrm{N}(3)$ & $142.29(11)$ \\
\hline $\mathrm{Si}(1)-\mathrm{C}(12)-\mathrm{H}(12 \mathrm{~A})$ & 109.5 & $\mathrm{~N}(3) \# 1-\operatorname{Zr}(1)-\mathrm{C}(9)$ & $100.12(9)$ \\
\hline $\mathrm{Si}(1)-\mathrm{C}(12)-\mathrm{H}(12 \mathrm{~B})$ & 109.5 & $\mathrm{~N}(3)-\operatorname{Zr}(1)-\mathrm{C}(9)$ & 103.97(9) \\
\hline $\mathrm{H}(12 \mathrm{~A})-\mathrm{C}(12)-\mathrm{H}(12 \mathrm{~B})$ & 109.5 & $\mathrm{~N}(3) \# 1-\operatorname{Zr}(1)-\mathrm{C}(9) \# 1$ & 103.97(9) \\
\hline $\mathrm{Si}(1)-\mathrm{C}(12)-\mathrm{H}(12 \mathrm{C})$ & 109.5 & $N(3)-Z r(1)-C(9) \# 1$ & $100.12(9)$ \\
\hline $\mathrm{H}(12 \mathrm{~A})-\mathrm{C}(12)-\mathrm{H}(12 \mathrm{C})$ & 109.5 & $C(9)-\operatorname{Zr}(1)-C(9) \# 1$ & $99.61(12)$ \\
\hline $\mathrm{H}(12 \mathrm{~B})-\mathrm{C}(12)-\mathrm{H}(12 \mathrm{C})$ & 109.5 & $\mathrm{~N}(3) \# 1-\operatorname{Zr}(1)-\mathrm{N}(6) \# 1$ & $73.72(7)$ \\
\hline $\mathrm{C}(4)-\mathrm{N}(3)-\mathrm{C}(2)$ & $113.50(19)$ & $\mathrm{N}(3)-\operatorname{Zr}(1)-\mathrm{N}(6) \# 1$ & $76.52(8)$ \\
\hline $\mathrm{C}(4)-\mathrm{N}(3)-\mathrm{Zr}(1)$ & $122.27(15)$ & $\mathrm{C}(9)-\mathrm{Zr}(1)-\mathrm{N}(6) \# 1$ & $92.80(8)$ \\
\hline $\mathrm{C}(2)-\mathrm{N}(3)-\mathrm{Zr}(1)$ & $118.04(16)$ & $\mathrm{C}(9) \# 1-\mathrm{Zr}(1)-\mathrm{N}(6) \# 1$ & $167.59(8)$ \\
\hline$C(5)-N(6)-C(8)$ & $112.4(2)$ & $\mathrm{N}(3) \# 1-\mathrm{Zr}(1)-\mathrm{N}(6)$ & $76.52(8)$ \\
\hline$C(5)-N(6)-C(7)$ & $109.0(2)$ & $\mathrm{N}(3)-\mathrm{Zr}(1)-\mathrm{N}(6)$ & $73.72(7)$ \\
\hline $\mathrm{C}(8)-\mathrm{N}(6)-\mathrm{C}(7)$ & $108.68(19)$ & $C(9)-\operatorname{Zr}(1)-\mathrm{N}(6)$ & $167.59(8)$ \\
\hline $\mathrm{C}(5)-\mathrm{N}(6)-\mathrm{Zr}(1)$ & $102.53(14)$ & $\mathrm{C}(9) \# 1-\mathrm{Zr}(1)-\mathrm{N}(6)$ & $92.80(8)$ \\
\hline $\mathrm{C}(8)-\mathrm{N}(6)-\mathrm{Zr}(1)$ & $110.94(15)$ & $\mathrm{N}(6) \# 1-\mathrm{Zr}(1)-\mathrm{N}(6)$ & $74.78(10)$ \\
\hline$C(7)-N(6)-Z r(1)$ & $113.23(14)$ & & \\
\hline
\end{tabular}

Symmetry transformations used to generate equivalent atoms: $\# 1 \quad-x+2, \quad y,-z+3 / 2$ 
Table S7. Anisotropic displacement parameters $\left(\AA^{2} \times 10^{3}\right)$ for $(\mathrm{CBC}) \mathrm{Zr}\left(\mathrm{CH}_{2} \mathrm{Si}\left(\mathrm{CH}_{3}\right)_{3}\right)_{2}$, 9.

$\begin{array}{llllll}\mathrm{U}_{11} & \mathrm{U}_{22} & \mathrm{U}_{33} & \mathrm{U}_{23} & \mathrm{U}_{13} & \mathrm{U}_{12}\end{array}$

\begin{tabular}{lllllll}
\hline $\mathrm{C}(1)$ & $18(1)$ & $22(1)$ & $22(1)$ & $1(1)$ & $-8(1)$ & $-2(1)$ \\
$\mathrm{C}(2)$ & $20(1)$ & $22(1)$ & $14(1)$ & $1(1)$ & $-5(1)$ & $-1(1)$ \\
$\mathrm{C}(4)$ & $24(1)$ & $26(1)$ & $15(1)$ & $-5(1)$ & $1(1)$ & $0(1)$ \\
$\mathrm{C}(5)$ & $23(1)$ & $20(1)$ & $21(1)$ & $-8(1)$ & $4(1)$ & $4(1)$ \\
$\mathrm{C}(7)$ & $14(1)$ & $19(1)$ & $25(1)$ & $-4(1)$ & $-2(1)$ & $4(1)$ \\
$\mathrm{C}(8)$ & $21(1)$ & $14(1)$ & $23(1)$ & $-1(1)$ & $-6(1)$ & $1(1)$ \\
$\mathrm{C}(9)$ & $14(1)$ & $17(1)$ & $22(1)$ & $3(1)$ & $1(1)$ & $1(1)$ \\
$\mathrm{C}(10)$ & $25(1)$ & $32(1)$ & $24(1)$ & $-10(1)$ & $5(1)$ & $-1(1)$ \\
$\mathrm{C}(11)$ & $24(1)$ & $25(1)$ & $35(2)$ & $-10(1)$ & $5(1)$ & $-2(1)$ \\
$\mathrm{C}(12)$ & $28(2)$ & $26(2)$ & $49(2)$ & $-5(1)$ & $7(1)$ & $10(1)$ \\
$\mathrm{N}(3)$ & $16(1)$ & $20(1)$ & $13(1)$ & $-1(1)$ & $1(1)$ & $0(1)$ \\
$\mathrm{N}(6)$ & $15(1)$ & $17(1)$ & $16(1)$ & $-3(1)$ & $-2(1)$ & $1(1)$ \\
$\mathrm{Si}(1)$ & $16(1)$ & $18(1)$ & $27(1)$ & $-4(1)$ & $3(1)$ & $2(1)$ \\
$\mathrm{Zr}(1)$ & $10(1)$ & $11(1)$ & $9(1)$ & 0 & $0(1)$ & 0 \\
& & & & & & \\
\hline
\end{tabular}

The anisotropic displacement factor exponent takes the form: $-2 \pi^{2}\left[h^{2} a * 2 U_{11}+\ldots+2 h k a^{*} b^{*} U_{12}\right]$ 
Table S8. Hydrogen coordinates $\left(\mathrm{x} 10^{4}\right)$ and isotropic displacement parameters $\left(\AA^{2} \times 10^{3}\right)$ for $(\mathrm{CBC}) \mathrm{Zr}\left(\mathrm{CH}_{2} \mathrm{Si}\left(\mathrm{CH}_{3}\right)_{3}\right)_{2}, 9$.

\begin{tabular}{|c|c|c|c|c|}
\hline & $\mathrm{x}$ & $\mathrm{y}$ & $\mathrm{z}$ & $\mathrm{U}(\mathrm{eq})$ \\
\hline $\mathrm{H}(1 \mathrm{~A})$ & 7415 & 1298 & 4681 & 27 \\
\hline $\mathrm{H}(1 \mathrm{~B})$ & 8278 & 770 & 4846 & 27 \\
\hline $\mathrm{H}(2 \mathrm{~A})$ & 8566 & 1828 & 3630 & 24 \\
\hline $\mathrm{H}(2 \mathrm{~B})$ & 8374 & 2319 & 4935 & 24 \\
\hline $\mathrm{H}(4 \mathrm{~A})$ & 10186 & 1412 & 3778 & 27 \\
\hline $\mathrm{H}(4 \mathrm{~B})$ & 9761 & 807 & 4676 & 27 \\
\hline $\mathrm{H}(5 \mathrm{~A})$ & 11316 & 709 & 5475 & 25 \\
\hline $\mathrm{H}(5 \mathrm{~B})$ & 11447 & 1569 & 5586 & 25 \\
\hline $\mathrm{H}(7 \mathrm{~A})$ & 12210 & 1624 & 7899 & 24 \\
\hline $\mathrm{H}(7 \mathrm{~B})$ & 12261 & 761 & 8005 & 24 \\
\hline $\mathrm{H}(8 \mathrm{~A})$ & 10748 & 361 & 8688 & 25 \\
\hline $\mathrm{H}(8 \mathrm{~B})$ & 10730 & 58 & 7097 & 25 \\
\hline $\mathrm{H}(9 \mathrm{~A})$ & 8793 & 3333 & 6944 & 21 \\
\hline $\mathrm{H}(9 \mathrm{~B})$ & 8295 & 2715 & 7674 & 21 \\
\hline $\mathrm{H}(10 \mathrm{~A})$ & 8122 & 2789 & 10732 & 40 \\
\hline $\mathrm{H}(10 \mathrm{~B})$ & 8752 & 3326 & 11834 & 40 \\
\hline $\mathrm{H}(10 \mathrm{C})$ & 9192 & 2625 & 11237 & 40 \\
\hline $\mathrm{H}(11 \mathrm{~A})$ & 10595 & 3559 & 10300 & 42 \\
\hline $\mathrm{H}(11 \mathrm{~B})$ & 10150 & 4259 & 10887 & 42 \\
\hline
\end{tabular}




\begin{tabular}{lrrrr}
$\mathrm{H}(11 \mathrm{C})$ & 10307 & 4239 & 9257 & 42 \\
$\mathrm{H}(12 \mathrm{~A})$ & 8312 & 4652 & 8363 & 52 \\
$\mathrm{H}(12 \mathrm{~B})$ & 8182 & 4608 & 10006 & 52 \\
$\mathrm{H}(12 \mathrm{C})$ & 7526 & 4151 & 8780 & 52 \\
\hline
\end{tabular}


Figure S4. ORTEP Drawing of $(\mathrm{CBC}) \mathrm{Zr}\left(\mathrm{C}_{4} \mathrm{Ph}_{4}\right), \mathbf{1 0}$.

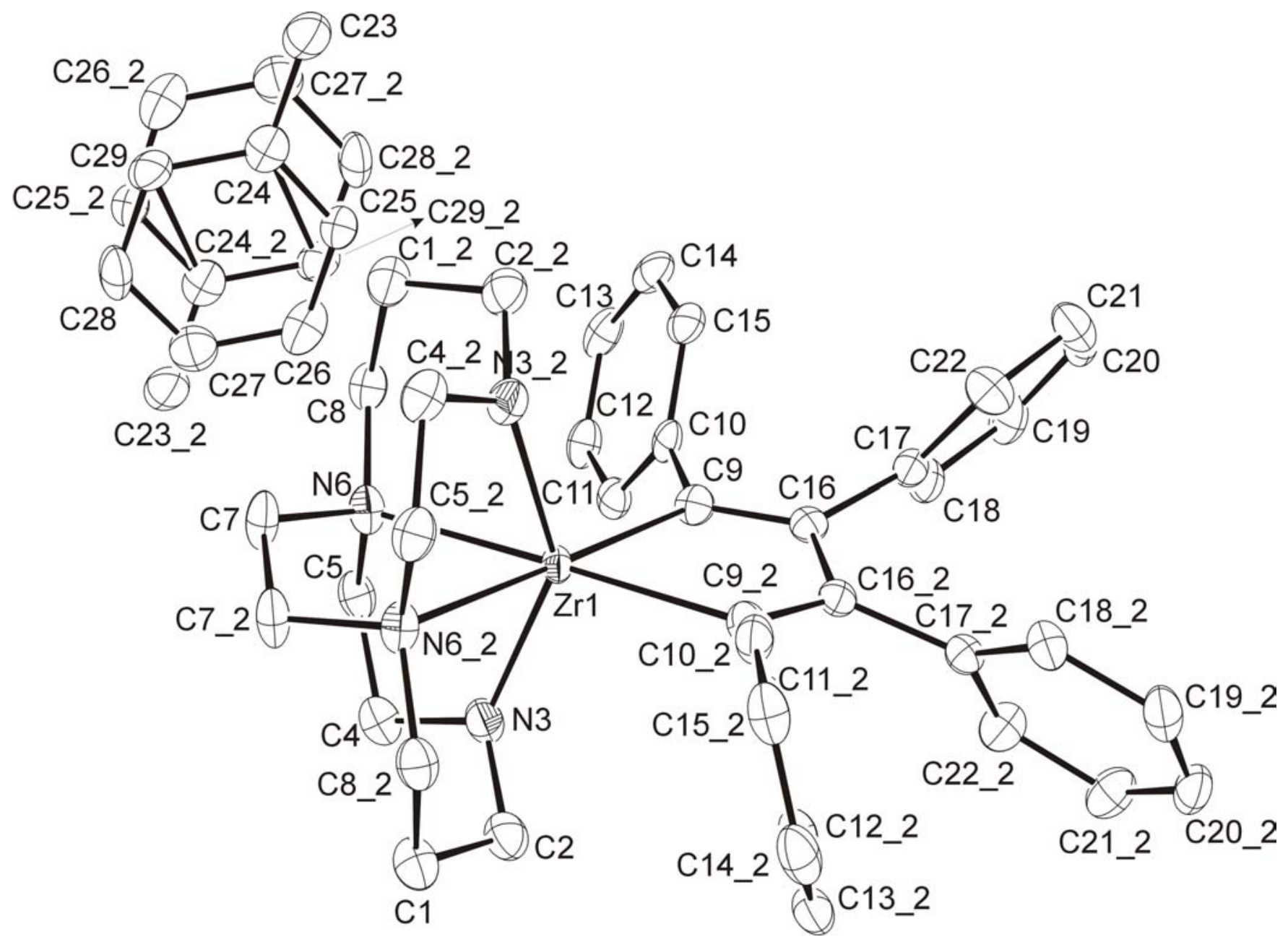


Table S9. Atomic coordinates $\left(\mathrm{x} 10^{4}\right)$ and equivalent isotropic displacement parameters $\left(\AA^{2} \times 10^{3}\right)$ for $(\mathrm{CBC}) \mathrm{Zr}\left(\mathrm{C}_{4} \mathrm{Ph}_{4}\right), \mathbf{1 0}$.

\begin{tabular}{|c|c|c|c|c|}
\hline & $\mathrm{x}$ & $\mathrm{y}$ & $\mathrm{z}$ & $\mathrm{U}(\mathrm{eq})$ \\
\hline$C(1)$ & $-301(1)$ & $3591(2)$ & $4247(1)$ & $28(1)$ \\
\hline$C(2)$ & $290(1)$ & $4499(2)$ & 4099(1) & $27(1)$ \\
\hline$C(4)$ & $1296(1)$ & $3582(2)$ & $3361(1)$ & $28(1)$ \\
\hline$C(5)$ & $1509(1)$ & $3432(2)$ & $2566(1)$ & $24(1)$ \\
\hline$C(7)$ & $295(2)$ & $2423(2)$ & 2190(1) & $28(1)$ \\
\hline$C(8)$ & $999(1)$ & $3501(2)$ & $1309(1)$ & $24(1)$ \\
\hline$C(9)$ & $726(1)$ & 6411(2) & 2091(1) & $20(1)$ \\
\hline$C(10)$ & $1430(1)$ & $6370(2)$ & $1617(1)$ & $20(1)$ \\
\hline$C(11)$ & $2168(1)$ & $6036(2)$ & 1915(1) & $22(1)$ \\
\hline$C(12)$ & $2835(1)$ & $5924(2)$ & $1485(1)$ & $26(1)$ \\
\hline$C(13)$ & $2783(1)$ & $6140(2)$ & $742(1)$ & $28(1)$ \\
\hline$C(14)$ & $2059(2)$ & $6466(2)$ & $437(1)$ & $27(1)$ \\
\hline$C(15)$ & 1392(1) & $6581(2)$ & $867(1)$ & $23(1)$ \\
\hline$C(16)$ & $354(1)$ & $7347(2)$ & $2251(1)$ & $18(1)$ \\
\hline$C(17)$ & $558(1)$ & $8390(2)$ & 1891(1) & $19(1)$ \\
\hline$C(18)$ & $1327(1)$ & $8817(2)$ & 1895(1) & $23(1)$ \\
\hline$C(19)$ & $1507(2)$ & $9722(2)$ & $1486(1)$ & $28(1)$ \\
\hline$C(20)$ & $924(2)$ & $10222(2)$ & $1059(1)$ & $29(1)$ \\
\hline
\end{tabular}




\begin{tabular}{lrrrr}
$\mathrm{C}(21)$ & $152(2)$ & $9827(2)$ & $1061(1)$ & $31(1)$ \\
$\mathrm{C}(22)$ & $-28(1)$ & $8936(2)$ & $1478(1)$ & $28(1)$ \\
$\mathrm{N}(3)$ & $675(1)$ & $4415(2)$ & $3399(1)$ & $25(1)$ \\
$\mathrm{N}(6)$ & $773(1)$ & $3423(1)$ & $2094(1)$ & $22(1)$ \\
$\mathrm{Zr}(1)$ & 0 & $4955(1)$ & 2500 & $15(1)$ \\
$\mathrm{C}(23)$ & $3349(5)$ & $1891(6)$ & $1038(4)$ & $36(2)$ \\
$\mathrm{C}(24)$ & $2852(3)$ & $2224(4)$ & $391(3)$ & $27(1)$ \\
$\mathrm{C}(25)$ & $2500(6)$ & $1498(9)$ & $-72(6)$ & $23(2)$ \\
$\mathrm{C}(26)$ & $2033(3)$ & $1789(5)$ & $-674(4)$ & $33(1)$ \\
$\mathrm{C}(27)$ & $1890(5)$ & $2863(6)$ & $-797(4)$ & $34(2)$ \\
$\mathrm{C}(28)$ & $2239(4)$ & $3633(4)$ & $-336(4)$ & $33(1)$ \\
$\mathrm{C}(29)$ & $2707(7)$ & $3322(9)$ & $267(6)$ & $31(2)$ \\
\hline
\end{tabular}

$\mathrm{U}(\mathrm{eq})$ is defined as one third of the trace of the orthogonalized $\mathrm{U}_{\mathrm{ij}}$ tensor. 
Table S10. Bond Lengths $(\AA)$ and Angles (deg) for $(\mathrm{CBC}) \mathrm{Zr}\left(\mathrm{C}_{4} \mathrm{Ph}_{4}\right), \mathbf{1 0}$.

\section{Bond Lengths}

\begin{tabular}{|c|c|c|c|}
\hline$C(1)-C(2)$ & $1.529(3)$ & $\mathrm{C}(8)-\mathrm{H}(8 \mathrm{~B})$ & 0.9900 \\
\hline $\mathrm{C}(1)-\mathrm{C}(8) \# 1$ & $1.536(3)$ & $C(9)-C(16)$ & $1.354(3)$ \\
\hline $\mathrm{C}(1)-\mathrm{H}(1 \mathrm{~A})$ & 0.9900 & $C(9)-C(10)$ & $1.480(3)$ \\
\hline $\mathrm{C}(1)-\mathrm{H}(1 \mathrm{~B})$ & 0.9900 & $C(9)-\operatorname{Zr}(1)$ & $2.313(2)$ \\
\hline $\mathrm{C}(2)-\mathrm{N}(3)$ & $1.449(3)$ & $C(10)-C(15)$ & $1.395(3)$ \\
\hline $\mathrm{C}(2)-\mathrm{H}(2 \mathrm{~A})$ & 0.9900 & $\mathrm{C}(10)-\mathrm{C}(11)$ & $1.402(3)$ \\
\hline $\mathrm{C}(2)-\mathrm{H}(2 \mathrm{~B})$ & 0.9900 & $C(11)-C(12)$ & $1.389(3)$ \\
\hline $\mathrm{C}(4)-\mathrm{N}(3)$ & $1.470(3)$ & $\mathrm{C}(11)-\mathrm{H}(11)$ & 0.9500 \\
\hline$C(4)-C(5)$ & $1.513(3)$ & $\mathrm{C}(12)-\mathrm{C}(13)$ & $1.384(3)$ \\
\hline $\mathrm{C}(4)-\mathrm{H}(4 \mathrm{~A})$ & 0.9900 & $\mathrm{C}(12)-\mathrm{H}(12)$ & 0.9500 \\
\hline $\mathrm{C}(4)-\mathrm{H}(4 \mathrm{~B})$ & 0.9900 & $C(13)-C(14)$ & $1.383(4)$ \\
\hline $\mathrm{C}(5)-\mathrm{N}(6)$ & $1.488(3)$ & $\mathrm{C}(13)-\mathrm{H}(13)$ & 0.9500 \\
\hline $\mathrm{C}(5)-\mathrm{H}(5 \mathrm{~A})$ & 0.9900 & $C(14)-C(15)$ & $1.388(3)$ \\
\hline $\mathrm{C}(5)-\mathrm{H}(5 \mathrm{~B})$ & 0.9900 & $\mathrm{C}(14)-\mathrm{H}(14)$ & 0.9500 \\
\hline $\mathrm{C}(7)-\mathrm{N}(6)$ & $1.491(3)$ & $\mathrm{C}(15)-\mathrm{H}(15)$ & 0.9500 \\
\hline$C(7)-C(7) \# 1$ & $1.520(5)$ & $C(16)-C(17)$ & $1.496(3)$ \\
\hline $\mathrm{C}(7)-\mathrm{H}(7 \mathrm{~A})$ & 0.9900 & $C(16)-C(16) \# 1$ & $1.511(4)$ \\
\hline $\mathrm{C}(7)-\mathrm{H}(7 \mathrm{~B})$ & 0.9900 & $\mathrm{C}(17)-\mathrm{C}(18)$ & $1.395(3)$ \\
\hline $\mathrm{C}(8)-\mathrm{N}(6)$ & $1.495(3)$ & $C(17)-C(22)$ & $1.398(3)$ \\
\hline $\mathrm{C}(8)-\mathrm{C}(1) \# 1$ & $1.536(3)$ & $\mathrm{C}(18)-\mathrm{C}(19)$ & $1.387(3)$ \\
\hline $\mathrm{C}(8)-\mathrm{H}(8 \mathrm{~A})$ & 0.9900 & $\mathrm{C}(18)-\mathrm{H}(18)$ & 0.9500 \\
\hline
\end{tabular}




\begin{tabular}{|c|c|c|c|}
\hline$C(19)-C(20)$ & $1.383(4)$ & $\mathrm{C}(28)-\mathrm{C}(29)$ & $1.392(9)$ \\
\hline C(19)-H(19) & 0.9500 & $\mathrm{C}(28)-\mathrm{H}(28 \mathrm{~A})$ & 0.9500 \\
\hline $\mathrm{C}(20)-\mathrm{C}(21)$ & $1.384(4)$ & C(29)-H(29A) & 0.9500 \\
\hline $\mathrm{C}(20)-\mathrm{H}(20)$ & 0.9500 & & \\
\hline $\mathrm{C}(21)-\mathrm{C}(22)$ & $1.380(3)$ & \multirow{2}{*}{\multicolumn{2}{|c|}{ Bond Angles }} \\
\hline $\mathrm{C}(21)-\mathrm{H}(21)$ & 0.9500 & & \\
\hline $\mathrm{C}(22)-\mathrm{H}(22)$ & 0.9500 & $C(2)-C(1)-C(8) \# 1$ & $115.13(19)$ \\
\hline $\mathrm{N}(3)-\operatorname{Zr}(1)$ & $2.0820(19)$ & $\mathrm{C}(2)-\mathrm{C}(1)-\mathrm{H}(1 \mathrm{~A})$ & 108.5 \\
\hline $\mathrm{N}(6)-\operatorname{Zr}(1)$ & $2.4269(19)$ & $\mathrm{C}(8) \# 1-\mathrm{C}(1)-\mathrm{H}(1 \mathrm{~A})$ & 108.5 \\
\hline $\mathrm{Zr}(1)-\mathrm{N}(3) \# 1$ & $2.0820(19)$ & $\mathrm{C}(2)-\mathrm{C}(1)-\mathrm{H}(1 \mathrm{~B})$ & 108.5 \\
\hline $\operatorname{Zr}(1)-C(9) \# 1$ & $2.313(2)$ & $\mathrm{C}(8) \# 1-\mathrm{C}(1)-\mathrm{H}(1 \mathrm{~B})$ & 108.5 \\
\hline $\mathrm{Zr}(1)-\mathrm{N}(6) \# 1$ & $2.4269(19)$ & $\mathrm{H}(1 \mathrm{~A})-\mathrm{C}(1)-\mathrm{H}(1 \mathrm{~B})$ & 107.5 \\
\hline$C(23)-C(24)$ & $1.487(8)$ & $\mathrm{N}(3)-\mathrm{C}(2)-\mathrm{C}(1)$ & $114.0(2)$ \\
\hline $\mathrm{C}(23)-\mathrm{H}(23 \mathrm{~A})$ & 0.9800 & $\mathrm{~N}(3)-\mathrm{C}(2)-\mathrm{H}(2 \mathrm{~A})$ & 108.8 \\
\hline $\mathrm{C}(23)-\mathrm{H}(23 \mathrm{~B})$ & 0.9800 & $\mathrm{C}(1)-\mathrm{C}(2)-\mathrm{H}(2 \mathrm{~A})$ & 108.8 \\
\hline $\mathrm{C}(23)-\mathrm{H}(23 \mathrm{C})$ & 0.9800 & $\mathrm{~N}(3)-\mathrm{C}(2)-\mathrm{H}(2 \mathrm{~B})$ & 108.8 \\
\hline$C(24)-C(25)$ & $1.362(12)$ & $\mathrm{C}(1)-\mathrm{C}(2)-\mathrm{H}(2 \mathrm{~B})$ & 108.8 \\
\hline C(24)-C(29) & $1.402(12)$ & $\mathrm{H}(2 \mathrm{~A})-\mathrm{C}(2)-\mathrm{H}(2 \mathrm{~B})$ & 107.6 \\
\hline$C(25)-C(26)$ & $1.381(9)$ & $\mathrm{N}(3)-\mathrm{C}(4)-\mathrm{C}(5)$ & $108.33(18)$ \\
\hline $\mathrm{C}(25)-\mathrm{H}(25 \mathrm{~A})$ & 0.9500 & $\mathrm{~N}(3)-\mathrm{C}(4)-\mathrm{H}(4 \mathrm{~A})$ & 110.0 \\
\hline$C(26)-C(27)$ & $1.373(10)$ & $\mathrm{C}(5)-\mathrm{C}(4)-\mathrm{H}(4 \mathrm{~A})$ & 110.0 \\
\hline $\mathrm{C}(26)-\mathrm{H}(26 \mathrm{~A})$ & 0.9500 & $\mathrm{~N}(3)-\mathrm{C}(4)-\mathrm{H}(4 \mathrm{~B})$ & 110.0 \\
\hline$C(27)-C(28)$ & $1.393(10)$ & $\mathrm{C}(5)-\mathrm{C}(4)-\mathrm{H}(4 \mathrm{~B})$ & 110.0 \\
\hline $\mathrm{C}(27)-\mathrm{H}(27 \mathrm{~A})$ & 0.9500 & $\mathrm{H}(4 \mathrm{~A})-\mathrm{C}(4)-\mathrm{H}(4 \mathrm{~B})$ & 108.4 \\
\hline
\end{tabular}




\begin{tabular}{|c|c|c|c|}
\hline $\mathrm{N}(6)-\mathrm{C}(5)-\mathrm{C}(4)$ & $110.16(18)$ & $C(12)-C(11)-C(10)$ & $121.8(2)$ \\
\hline $\mathrm{N}(6)-\mathrm{C}(5)-\mathrm{H}(5 \mathrm{~A})$ & 109.6 & $\mathrm{C}(12)-\mathrm{C}(11)-\mathrm{H}(11)$ & 119.1 \\
\hline $\mathrm{C}(4)-\mathrm{C}(5)-\mathrm{H}(5 \mathrm{~A})$ & 109.6 & $\mathrm{C}(10)-\mathrm{C}(11)-\mathrm{H}(11)$ & 119.1 \\
\hline $\mathrm{N}(6)-\mathrm{C}(5)-\mathrm{H}(5 \mathrm{~B})$ & 109.6 & $C(13)-C(12)-C(11)$ & $120.0(2)$ \\
\hline $\mathrm{C}(4)-\mathrm{C}(5)-\mathrm{H}(5 \mathrm{~B})$ & 109.6 & $\mathrm{C}(13)-\mathrm{C}(12)-\mathrm{H}(12)$ & 120.0 \\
\hline $\mathrm{H}(5 \mathrm{~A})-\mathrm{C}(5)-\mathrm{H}(5 \mathrm{~B})$ & 108.1 & $\mathrm{C}(11)-\mathrm{C}(12)-\mathrm{H}(12)$ & 120.0 \\
\hline$N(6)-C(7)-C(7) \# 1$ & $116.62(15)$ & $C(14)-C(13)-C(12)$ & $119.1(2)$ \\
\hline N(6)-C(7)-H(7A) & 108.1 & $\mathrm{C}(14)-\mathrm{C}(13)-\mathrm{H}(13)$ & 120.5 \\
\hline $\mathrm{C}(7) \# 1-\mathrm{C}(7)-\mathrm{H}(7 \mathrm{~A})$ & 108.1 & $\mathrm{C}(12)-\mathrm{C}(13)-\mathrm{H}(13)$ & 120.5 \\
\hline $\mathrm{N}(6)-\mathrm{C}(7)-\mathrm{H}(7 \mathrm{~B})$ & 108.1 & $C(13)-C(14)-C(15)$ & $121.0(2)$ \\
\hline $\mathrm{C}(7) \# 1-\mathrm{C}(7)-\mathrm{H}(7 \mathrm{~B})$ & 108.1 & $\mathrm{C}(13)-\mathrm{C}(14)-\mathrm{H}(14)$ & 119.5 \\
\hline $\mathrm{H}(7 \mathrm{~A})-\mathrm{C}(7)-\mathrm{H}(7 \mathrm{~B})$ & 107.3 & $\mathrm{C}(15)-\mathrm{C}(14)-\mathrm{H}(14)$ & 119.5 \\
\hline $\mathrm{N}(6)-\mathrm{C}(8)-\mathrm{C}(1) \# 1$ & $115.55(19)$ & $C(14)-C(15)-C(10)$ & $121.1(2)$ \\
\hline $\mathrm{N}(6)-\mathrm{C}(8)-\mathrm{H}(8 \mathrm{~A})$ & 108.4 & $\mathrm{C}(14)-\mathrm{C}(15)-\mathrm{H}(15)$ & 119.5 \\
\hline $\mathrm{C}(1) \# 1-\mathrm{C}(8)-\mathrm{H}(8 \mathrm{~A})$ & 108.4 & $\mathrm{C}(10)-\mathrm{C}(15)-\mathrm{H}(15)$ & 119.5 \\
\hline $\mathrm{N}(6)-\mathrm{C}(8)-\mathrm{H}(8 \mathrm{~B})$ & 108.4 & $C(9)-C(16)-C(17)$ & $122.51(19)$ \\
\hline $\mathrm{C}(1) \# 1-\mathrm{C}(8)-\mathrm{H}(8 \mathrm{~B})$ & 108.4 & $C(9)-C(16)-C(16) \# 1$ & $120.13(13)$ \\
\hline $\mathrm{H}(8 \mathrm{~A})-\mathrm{C}(8)-\mathrm{H}(8 \mathrm{~B})$ & 107.5 & $C(17)-C(16)-C(16) \# 1$ & $117.05(12)$ \\
\hline$C(16)-C(9)-C(10)$ & $122.24(19)$ & $\mathrm{C}(18)-\mathrm{C}(17)-\mathrm{C}(22)$ & $117.2(2)$ \\
\hline $\mathrm{C}(16)-\mathrm{C}(9)-\mathrm{Zr}(1)$ & $110.62(15)$ & $C(18)-C(17)-C(16)$ & $123.19(19)$ \\
\hline $\mathrm{C}(10)-\mathrm{C}(9)-\operatorname{Zr}(1)$ & $126.47(15)$ & $C(22)-C(17)-C(16)$ & $119.46(19)$ \\
\hline$C(15)-C(10)-C(11)$ & $117.1(2)$ & $\mathrm{C}(19)-\mathrm{C}(18)-\mathrm{C}(17)$ & $121.2(2)$ \\
\hline$C(15)-C(10)-C(9)$ & $123.3(2)$ & $\mathrm{C}(19)-\mathrm{C}(18)-\mathrm{H}(18)$ & 119.4 \\
\hline$C(11)-C(10)-C(9)$ & $119.5(2)$ & $\mathrm{C}(17)-\mathrm{C}(18)-\mathrm{H}(18)$ & 119.4 \\
\hline
\end{tabular}




\begin{tabular}{|c|c|c|c|}
\hline$C(20)-C(19)-C(18)$ & $120.5(2)$ & $\mathrm{N}(3) \# 1-\operatorname{Zr}(1)-\mathrm{C}(9) \# 1$ & $102.99(8)$ \\
\hline $\mathrm{C}(20)-\mathrm{C}(19)-\mathrm{H}(19)$ & 119.7 & $\mathrm{~N}(3)-\mathrm{Zr}(1)-\mathrm{C}(9) \# 1$ & $106.17(8)$ \\
\hline $\mathrm{C}(18)-\mathrm{C}(19)-\mathrm{H}(19)$ & 119.7 & $\mathrm{C}(9)-\mathrm{Zr}(1)-\mathrm{C}(9) \# 1$ & $77.19(11)$ \\
\hline$C(19)-C(20)-C(21)$ & $119.2(2)$ & $\mathrm{N}(3) \# 1-\mathrm{Zr}(1)-\mathrm{N}(6)$ & $78.00(7)$ \\
\hline $\mathrm{C}(19)-\mathrm{C}(20)-\mathrm{H}(20)$ & 120.4 & $\mathrm{~N}(3)-\mathrm{Zr}(1)-\mathrm{N}(6)$ & $72.74(7)$ \\
\hline $\mathrm{C}(21)-\mathrm{C}(20)-\mathrm{H}(20)$ & 120.4 & $\mathrm{C}(9)-\mathrm{Zr}(1)-\mathrm{N}(6)$ & $103.01(7)$ \\
\hline$C(22)-C(21)-C(20)$ & $120.1(2)$ & $\mathrm{C}(9) \# 1-\mathrm{Zr}(1)-\mathrm{N}(6)$ & 178.91(7) \\
\hline $\mathrm{C}(22)-\mathrm{C}(21)-\mathrm{H}(21)$ & 119.9 & $\mathrm{~N}(3) \# 1-\mathrm{Zr}(1)-\mathrm{N}(6) \# 1$ & $72.74(7)$ \\
\hline $\mathrm{C}(20)-\mathrm{C}(21)-\mathrm{H}(21)$ & 119.9 & $\mathrm{~N}(3)-\mathrm{Zr}(1)-\mathrm{N}(6) \# 1$ & $78.00(7)$ \\
\hline $\mathrm{C}(21)-\mathrm{C}(22)-\mathrm{C}(17)$ & $121.8(2)$ & $\mathrm{C}(9)-\mathrm{Zr}(1)-\mathrm{N}(6) \# 1$ & 178.91(7) \\
\hline $\mathrm{C}(21)-\mathrm{C}(22)-\mathrm{H}(22)$ & 119.1 & $\mathrm{C}(9) \# 1-\mathrm{Zr}(1)-\mathrm{N}(6) \# 1$ & 103.01(7) \\
\hline $\mathrm{C}(17)-\mathrm{C}(22)-\mathrm{H}(22)$ & 119.1 & $\mathrm{~N}(6)-\operatorname{Zr}(1)-\mathrm{N}(6) \# 1$ & 76.81(9) \\
\hline $\mathrm{C}(2)-\mathrm{N}(3)-\mathrm{C}(4)$ & $114.96(18)$ & $C(25)-C(24)-C(29)$ & $118.2(6)$ \\
\hline $\mathrm{C}(2)-\mathrm{N}(3)-\mathrm{Zr}(1)$ & $115.23(14)$ & $C(25)-C(24)-C(23)$ & $122.4(7)$ \\
\hline $\mathrm{C}(4)-\mathrm{N}(3)-\mathrm{Zr}(1)$ & $124.24(15)$ & $C(29)-C(24)-C(23)$ & $119.3(7)$ \\
\hline $\mathrm{C}(5)-\mathrm{N}(6)-\mathrm{C}(7)$ & $112.26(18)$ & $C(24)-C(25)-C(26)$ & 123.3(9) \\
\hline $\mathrm{C}(5)-\mathrm{N}(6)-\mathrm{C}(8)$ & $109.21(17)$ & $\mathrm{C}(24)-\mathrm{C}(25)-\mathrm{H}(25 \mathrm{~A})$ & 118.3 \\
\hline $\mathrm{C}(7)-\mathrm{N}(6)-\mathrm{C}(8)$ & $108.36(17)$ & $\mathrm{C}(26)-\mathrm{C}(25)-\mathrm{H}(25 \mathrm{~A})$ & 118.3 \\
\hline $\mathrm{C}(5)-\mathrm{N}(6)-\mathrm{Zr}(1)$ & $105.05(12)$ & $C(27)-C(26)-C(25)$ & $118.6(7)$ \\
\hline $\mathrm{C}(7)-\mathrm{N}(6)-\mathrm{Zr}(1)$ & $108.95(13)$ & $\mathrm{C}(27)-\mathrm{C}(26)-\mathrm{H}(26 \mathrm{~A})$ & 120.7 \\
\hline $\mathrm{C}(8)-\mathrm{N}(6)-\mathrm{Zr}(1)$ & $113.05(13)$ & $\mathrm{C}(25)-\mathrm{C}(26)-\mathrm{H}(26 \mathrm{~A})$ & 120.7 \\
\hline $\mathrm{N}(3) \# 1-\operatorname{Zr}(1)-\mathrm{N}(3)$ & $142.43(10)$ & $C(26)-C(27)-C(28)$ & $119.9(7)$ \\
\hline N(3)\#1-Zr(1)-C(9) & $106.17(8)$ & $\mathrm{C}(26)-\mathrm{C}(27)-\mathrm{H}(27 \mathrm{~A})$ & 120.0 \\
\hline N(3)-Zr(1)-C(9) & $102.99(8)$ & $\mathrm{C}(28)-\mathrm{C}(27)-\mathrm{H}(27 \mathrm{~A})$ & 120.0 \\
\hline
\end{tabular}




$\begin{array}{llll}\mathrm{C}(29)-\mathrm{C}(28)-\mathrm{C}(27) & 120.5(8) & \mathrm{C}(28)-\mathrm{C}(29)-\mathrm{H}(29 \mathrm{~A}) & 120.3 \\ \mathrm{C}(29)-\mathrm{C}(28)-\mathrm{H}(28 \mathrm{~A}) & 119.8 & \mathrm{C}(24)-\mathrm{C}(29)-\mathrm{H}(29 \mathrm{~A}) & 120.3 \\ \mathrm{C}(27)-\mathrm{C}(28)-\mathrm{H}(28 \mathrm{~A}) & 119.8 & & \\ \mathrm{C}(28)-\mathrm{C}(29)-\mathrm{C}(24) & 119.4(10) & & \end{array}$

Symmetry transformations used to generate equivalent atoms: \#1 -x, y, -z+1/2 
Table S11. Anisotropic displacement parameters $\left(\AA^{2} \times 10^{3}\right)$ for $(\mathrm{CBC}) \mathrm{Zr}_{(}\left(\mathrm{C}_{4} \mathrm{Ph}_{4}\right), \mathbf{1 0}$.

$$
\begin{array}{llllll}
\mathrm{U}_{11} & \mathrm{U}_{22} & \mathrm{U}_{33} & \mathrm{U}_{23} & \mathrm{U}_{13} & \mathrm{U}_{12}
\end{array}
$$

\begin{tabular}{|c|c|c|c|c|c|c|}
\hline$C(1)$ & $28(1)$ & $24(1)$ & $33(1)$ & 3(1) & $-2(1)$ & $-1(1)$ \\
\hline$C(2)$ & $27(1)$ & $24(1)$ & $29(1)$ & $3(1)$ & $-3(1)$ & 2(1) \\
\hline $\mathrm{C}(4)$ & $27(1)$ & $24(1)$ & $32(1)$ & $5(1)$ & 1(1) & 2(1) \\
\hline C(5) & 19(1) & $20(1)$ & $33(1)$ & $4(1)$ & 1(1) & $4(1)$ \\
\hline$C(7)$ & $28(1)$ & $13(1)$ & $42(2)$ & $-2(1)$ & $8(1)$ & $-2(1)$ \\
\hline$C(8)$ & $22(1)$ & $20(1)$ & $31(1)$ & $-3(1)$ & $3(1)$ & 1(1) \\
\hline $\mathrm{C}(9)$ & 19(1) & $20(1)$ & $22(1)$ & $-1(1)$ & $0(1)$ & $-3(1)$ \\
\hline $\mathrm{C}(10)$ & $23(1)$ & 11(1) & $26(1)$ & $-4(1)$ & $3(1)$ & $-5(1)$ \\
\hline$C(11)$ & $24(1)$ & $16(1)$ & $25(1)$ & $0(1)$ & $3(1)$ & $-3(1)$ \\
\hline$C(12)$ & 21(1) & $18(1)$ & $39(1)$ & $-5(1)$ & $3(1)$ & $-3(1)$ \\
\hline$C(13)$ & $26(1)$ & $23(1)$ & $36(1)$ & $-10(1)$ & 13(1) & $-9(1)$ \\
\hline$C(14)$ & $36(1)$ & $22(1)$ & $24(1)$ & $-6(1)$ & $6(1)$ & $-11(1)$ \\
\hline$C(15)$ & $26(1)$ & $18(1)$ & $25(1)$ & $-4(1)$ & $-2(1)$ & $-5(1)$ \\
\hline$C(16)$ & $17(1)$ & $19(1)$ & $18(1)$ & $0(1)$ & $-4(1)$ & $-2(1)$ \\
\hline$C(17)$ & $20(1)$ & $18(1)$ & $20(1)$ & $-2(1)$ & 1(1) & 1(1) \\
\hline$C(18)$ & $23(1)$ & $19(1)$ & $29(1)$ & $-3(1)$ & $-2(1)$ & $-1(1)$ \\
\hline$C(19)$ & $29(1)$ & 19(1) & $36(1)$ & $-4(1)$ & $7(1)$ & $-5(1)$ \\
\hline$C(20)$ & $43(2)$ & $15(1)$ & $29(1)$ & 2(1) & 11(1) & $0(1)$ \\
\hline$C(21)$ & $33(1)$ & $28(1)$ & $33(1)$ & 10(1) & 1(1) & $6(1)$ \\
\hline$C(22)$ & $22(1)$ & $30(1)$ & $31(1)$ & $6(1)$ & $-1(1)$ & $-1(1)$ \\
\hline
\end{tabular}




$\begin{array}{ccccccc}\mathrm{N}(3) & 29(1) & 19(1) & 27(1) & 3(1) & -3(1) & 1(1) \\ \mathrm{N}(6) & 19(1) & 17(1) & 31(1) & 1(1) & 4(1) & 0(1) \\ \mathrm{Zr}(1) & 15(1) & 11(1) & 18(1) & 0 & -2(1) & 0 \\ \mathrm{C}(23) & 52(5) & 31(4) & 27(4) & -2(3) & 10(3) & -10(3) \\ \mathrm{C}(24) & 24(3) & 28(3) & 30(3) & -2(2) & 10(2) & -5(2) \\ \mathrm{C}(25) & 19(5) & 21(4) & 28(6) & -2(4) & 1(4) & -1(3) \\ \mathrm{C}(26) & 18(3) & 33(3) & 49(4) & -13(3) & -2(3) & -1(2) \\ \mathrm{C}(27) & 36(4) & 36(4) & 31(5) & 0(3) & 5(3) & 1(3) \\ \mathrm{C}(28) & 44(4) & 16(3) & 40(4) & 4(3) & 17(3) & -1(2) \\ \mathrm{C}(29) & 40(7) & 27(5) & 25(5) & -3(4) & -3(4) & -11(4)\end{array}$

The anisotropic displacement factor exponent takes the form: $-2 \pi^{2}\left[h^{2} a^{* 2} U_{11}+\ldots+2 h\right.$ $\left.\mathrm{k} \mathrm{a} * \mathrm{~b} * \mathrm{U}_{12}\right]$ 
Table S12. Hydrogen coordinates $\left(\mathrm{x} 10^{4}\right)$ and isotropic displacement parameters $\left(\AA^{2} \mathrm{x}\right.$ $\left.10^{3}\right)$ for $(\mathrm{CBC}) \mathrm{Zr}\left(\mathrm{C}_{4} \mathrm{Ph}_{4}\right), \mathbf{1 0}$.

\begin{tabular}{|c|c|c|c|c|}
\hline & $\mathrm{x}$ & $\mathrm{y}$ & $\mathrm{z}$ & $\mathrm{U}(\mathrm{eq})$ \\
\hline $\mathrm{H}(1 \mathrm{~A})$ & -520 & 3698 & 4741 & 34 \\
\hline $\mathrm{H}(1 \mathrm{~B})$ & -7 & 2899 & 4254 & 34 \\
\hline $\mathrm{H}(2 \mathrm{~A})$ & 5 & 5195 & 4120 & 32 \\
\hline $\mathrm{H}(2 \mathrm{~B})$ & 706 & 4501 & 4494 & 32 \\
\hline $\mathrm{H}(4 \mathrm{~A})$ & 1097 & 2896 & 3565 & 33 \\
\hline $\mathrm{H}(4 \mathrm{~B})$ & 1773 & 3805 & 3652 & 33 \\
\hline $\mathrm{H}(5 \mathrm{~A})$ & 1863 & 4024 & 2413 & 29 \\
\hline $\mathrm{H}(5 \mathrm{~B})$ & 1799 & 2744 & 2509 & 29 \\
\hline $\mathrm{H}(7 \mathrm{~A})$ & 669 & 1815 & 2270 & 33 \\
\hline $\mathrm{H}(7 \mathrm{~B})$ & -4 & 2281 & 1726 & 33 \\
\hline $\mathrm{H}(8 \mathrm{~A})$ & 1317 & 2856 & 1186 & 29 \\
\hline $\mathrm{H}(8 \mathrm{~B})$ & 1348 & 4137 & 1252 & 29 \\
\hline $\mathrm{H}(11)$ & 2212 & 5882 & 2424 & 26 \\
\hline $\mathrm{H}(12)$ & 3328 & 5699 & 1702 & 31 \\
\hline $\mathrm{H}(13)$ & 3237 & 6066 & 444 & 34 \\
\hline $\mathrm{H}(14)$ & 2018 & 6614 & -73 & 33 \\
\hline $\mathrm{H}(15)$ & 902 & 6808 & 645 & 27 \\
\hline $\mathrm{H}(18)$ & 1735 & 8481 & 2184 & 28 \\
\hline $\mathrm{H}(19)$ & 2035 & 10000 & 1498 & 34 \\
\hline
\end{tabular}




\begin{tabular}{lcccc}
$\mathrm{H}(20)$ & 1051 & 10830 & 768 & 35 \\
$\mathrm{H}(21)$ & -255 & 10170 & 776 & 38 \\
$\mathrm{H}(22)$ & -563 & 8685 & 1484 & 33 \\
$\mathrm{H}(23 \mathrm{~A})$ & 3292 & 1114 & 1116 & 54 \\
$\mathrm{H}(23 \mathrm{~B})$ & 3174 & 2279 & 1473 & 54 \\
$\mathrm{H}(23 \mathrm{C})$ & 3910 & 2061 & 951 & 54 \\
$\mathrm{H}(25 \mathrm{~A})$ & 2580 & 753 & 22 & 27 \\
$\mathrm{H}(26 \mathrm{~A})$ & 1816 & 1258 & -995 & 40 \\
$\mathrm{H}(27 \mathrm{~A})$ & 1553 & 3081 & -1196 & 41 \\
$\mathrm{H}(28 \mathrm{~A})$ & 2156 & 4377 & -434 & 40 \\
$\mathrm{H}(29 \mathrm{~A})$ & 2926 & 3848 & 592 & 37 \\
\hline
\end{tabular}

